\title{
Solving the linearized forward-speed radiation problem using a high-order finite difference method on overlapping grids
}

\author{
Amini Afshar, Mostafa; Bingham, Harry B.
}

\section{Published in:}

Applied Ocean Research

Link to article, DOI:

10.1016/j.apor.2017.11.001

Publication date:

2017

Document Version

Peer reviewed version

Link back to DTU Orbit

Citation $(A P A)$ :

Amini Afshar, M., \& Bingham, H. B. (2017). Solving the linearized forward-speed radiation problem using a highorder finite difference method on overlapping grids. Applied Ocean Research, 69, 220-244.

https://doi.org/10.1016/j.apor.2017.11.001

\section{General rights}

Copyright and moral rights for the publications made accessible in the public portal are retained by the authors and/or other copyright owners and it is a condition of accessing publications that users recognise and abide by the legal requirements associated with these rights.

- Users may download and print one copy of any publication from the public portal for the purpose of private study or research.

- You may not further distribute the material or use it for any profit-making activity or commercial gain

- You may freely distribute the URL identifying the publication in the public portal 


\title{
Solving the linearized forward-speed radiation problem using a high-order finite difference method on overlapping grids
}

\author{
Mostafa Amini Afshar and Harry B. Bingham \\ Technical University of Denmark \\ Department of Mechanical Engineering \\ Section for Fluid Mechanics, Coastal \& Maritime Engineering \\ maaf@mek.dtu .dk, hbb@mek.dtu .dk
}

\begin{abstract}
The linearized potential flow approximation for the forward speed radiation problem is solved in the time domain using a high-order finite difference method. The finite-difference discretization is developed on overlapping, curvilinear body-fitted grids. To ensure numerical stability, the convective derivatives in the free-surface boundary conditions are treated using an upwind-biased stencil. Instead of solving for the radiation impulse response functions, a pseudo-impulsive Gaussian type displacement is employed in order to tailor the frequency-content to the discrete spatial resolution. Frequencydomain results are then obtained from a Fourier transform of the force and motion signals. In order to make a robust Fourier transform, and capture the response around the critical frequency, the tail of the force signal is asymptotically extrapolated assuming a linear decay rate. Fourth-order convergence of the calculations on simple geometries is demonstrated, along with a nearly linear scaling of the solution effort with increasing grid resolution. The code is validated by comparison with analytical and semi-analytical solutions using submerged and floating closed-form geometries. Calculations are also made for a modern bulk carrier, and good agreement is found with experimental measurements.
\end{abstract}

\section{Keywords}

Forward-speed radiation, Double-body, Generalized m-terms, Finite difference, Overlapping grids

\section{Introduction}

The problem of wave structure interaction has been solved for many years using a linearized potential flow approximation. Considering the size of large marine structures like ships, the use of potential flow theory is justified by the inherently large Reynolds numbers which mostly confine the effects of viscosity to thin boundary layers. The assumption of linearity is also acceptable as long as the oscillatory motions of the structure and the wave amplitudes are small, such that the first order terms in the perturbation expansion of the total nonlinear solution still capture a significant part of the physics of the problem. The problem is thus decomposed into steady, radiation and diffraction problems, each of which can be solved independently.

Traditionally, numerical solutions to these hydrodynamic problems, either in the frequency- or in the time-domain, are sought through the application of the boundary element method, where only some or all of the fluid boundary surfaces need to be discretized. WAMIT [1] can be mentioned as a well-known example of a frequency-domain solver for the wave-structure interaction problems with zero forward speed. Among the time-domain studies of wave-structure interaction and ship motions, one can for example refer to [2], [3], [4], [5], [6] and [7]. 
At the Technical University of Denmark (DTU), a new computational framework based on the highorder finite-difference method has been under development over the past decade for the simulation of water waves and wave-structure interaction [8], [9] and [10]. The use of the finite-difference method for the numerical approximation of the hydrodynamic problems requires that the whole computational domain be discretized. In comparison with the boundary element method, this strategy will generally require solving a larger system of equations. The system of equations in the case of the boundary element method is however a fully dense matrix, as basically each point would take influence from all other points in the discretized domain. Sparsification techniques have been developed based either on multipole expansions or pre-corrected FFT methods, see for example [11, 12], but these methods have so far not been applied to seakeeping calculations. The finite difference method, on the other hand, leads naturally to a very sparse system of equations, as the approximation for a derivative at one point depends only on a small number of the surrounding grid points. As a result, the finite-difference methodology can readily produce an optimal linear scaling of the computational effort with increasing number of grid points [9] and [10].

As part of this ongoing development, a new project was started at DTU aiming to develop a linearized potential flow seakeeping solver based on the high-order finite-difference discretization scheme [13]. This project also aimed to calculate the second-order wave drift forces from the computed first order results. A convincing demonstration of convergence of the wave drift forces for sailing ships using the boundary element method has so far been elusive. However, we anticipate that the better scaling properties of the finite-difference method could make such a demonstration possible. This goal has been achieved for the simple Wigley hull geometry [14] and work is in progress with more realistic hull forms.

The goal of this paper is to demonstrate that the adopted numerical solution method achieves high-order (4th-order) convergence under spatial grid refinement, along with a linear scaling of the solution effort with increasing number of grid points. These features make the method competitive with Rankine-type BEM solutions. We focus here on the radiation problem, while the steady and diffraction problems will be presented in follow-up papers. The problem is solved in the time domain. In the original time-domain approach, the body boundary condition is prescribed as an impulsive displacement, velocity or acceleration [5], [15]. The resulting radiation impulse response functions, can then be Fourier transformed to give the frequency-domain hydrodynamic coefficients. As first described by [16], another option is to prescribe a smooth pseudo-impulsive motion of the body using for example a Gaussian shape function, and this is the approach adopted here. The velocity potential in the field induced by this pseudo-impulsive motion is calculated at each time step and the solution is evolved forward in time by integration of the dynamic and kinematic free surface conditions. Forces on the body are calculated by integrating the pressures over the surface of the body, and frequencydomain results are then computed by Fourier transform of the time domain quantities. Asymptotic continuation is applied to capture the oscillation due to the critical frequency, $\omega_{c}\left(\tau=\omega_{c} U / g=1 / 4\right.$, $U$ the ship speed and $g$ gravitational acceleration) in the manner described by [17].

The formulation of the problem is reviewed in the next section, where both the Neumann-Kelvin and the double body linearizations are considered. The numerical solution strategy is described in Section 3, with particular emphasis on stable schemes for computing the convective derivatives in the free surface boundary conditions. Section 4 describes the design of the pseudo-impulsive, timedomain solution, while Section 5 details the treatment of the critical frequency and subsequent Fourier transform to obtain the frequency-domain solutions. Results are presented in Section 6 including demonstration of convergence and the scaling of the solution effort, and validation cases using closedform geometries with comparison to analytical solutions. Some detail about the hyperbolic grid generation is also given in Section 6, along with a comparison of the hydrodynamic coefficients for a real ship geometry with experimental measurements. 


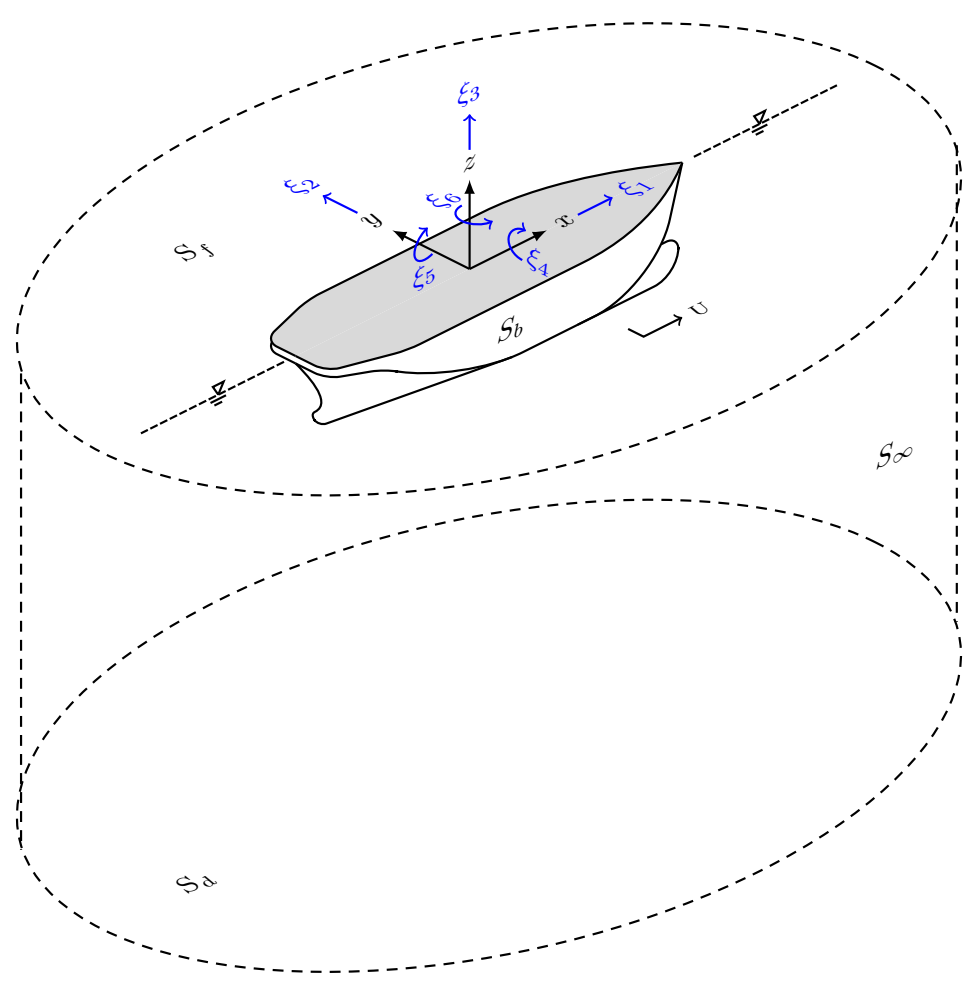

Figure 1: The sketch of the moving body and the flow domain.

\section{The mathematical formulation}

In this section we review the mathematical formulation for describing the motion of the body moving with a forward speed $U$ and oscillating at or near the free surface [18]. The formulation is based on potential flow theory. The body is assumed rigid and can oscillate in six degrees of freedom. Three translational modes are defined as surge $\xi_{1}$, sway $\xi_{2}$ and heave $\xi_{3}$ along the $x, y$ and $z$ axes respectively. Around these axes are also defined three rotational modes as roll $\xi_{4}=\Omega_{1}$, pitch $\xi_{5}=\Omega_{2}$ and yaw $\xi_{6}=\Omega_{3}$, see Figure 1. Three right-handed orthogonal coordinate systems are adopted. The first system $\left(O^{\prime}-x^{\prime} y^{\prime} z^{\prime}\right)$, is fixed to the body and oscillates and moves as the body does. The $x^{\prime}$ coordinate is along the length of the ship and $z^{\prime}$ is vertically upward through the center of gravity of the ship. The origin $O^{\prime}$ is also located in the plane of the undisturbed free surface. The second coordinate system $(O-x y z)$ is a steady-moving reference frame, and just translates with the constant forward speed $U$. At time $t=0$ these two coordinate systems coincide. The third coordinate system is earth-fixed and is denoted by $\left(O_{0}-x_{0}, y_{0}, z_{0}\right)$. The velocity potential in the domain can be defined by $\Phi\left(x_{0}, y_{0}, z_{0}, t\right)$.

\subsection{Linear formulation}

The linear formulation can be obtained by linearization of the exact nonlinear problem which is briefly reviewed in Appendix A. The nonlinear velocity potential in the moving coordinate system given by $\bar{\phi}$ can be expressed as:

$$
\Phi\left(x_{0}, y_{0}, z_{0}, t\right)=\Phi(x+U t, y, z, t) \equiv \bar{\phi}(x, y, z, t) .
$$

The nonlinear formulation is linearized first by assuming that $\bar{\phi}$ can be decomposed up to the first order as follows:

$$
\bar{\phi}(x, y, z, t)=\phi_{b}(x, y, z)+\phi(x, y, z, t) .
$$


The first order velocity potential is denoted by $\phi$ which satisfies the continuity equation as:

$$
\nabla^{2} \phi=\frac{\partial^{2} \phi}{\partial x^{2}}+\frac{\partial^{2} \phi}{\partial y^{2}}+\frac{\partial^{2} \phi}{\partial z^{2}}
$$

The time independent steady base flow is defined by the zeroth order velocity potential $\phi_{b}$. The base flow velocity potential is in fact describing the steady flow field with respect to the moving frame of reference when there is no oscillatory body motion. The steady potential can also be written as:

$$
\phi_{b}(x, y, z)=-U x+\phi_{b}^{\prime}(x, y, z)
$$

where in the simplest case, i.e. the Neumann-Kelvin linearization, $\phi_{b}^{\prime}$ is set to zero and the resulting base flow will be just a uniform flow described by $-U x$. Another widely adopted approximation is to take $\phi_{b}^{\prime}$ to be the double-body flow $\phi_{d b}$, which can be computed by solving the following boundary value problem:

$$
\begin{array}{rlrl}
\nabla^{2} \phi_{d b} & =0, & \\
\frac{\partial \phi_{d b}}{\partial n}=\boldsymbol{W} \cdot \boldsymbol{n} & & \text { on } S_{b}, \\
\frac{\partial \phi_{d b}}{\partial z} & =0 & & \text { on } z=0, \\
\nabla \phi_{d b} & \rightarrow 0 & & \text { in the far field }\left(S_{\infty}\right),
\end{array}
$$

where $\boldsymbol{W}=(U, 0,0)$. This boundary value problem describes an infinite domain flow around a combination of the body and its mirror image with respect to the $z=0$ plane. The linearization using this infinite fluid flow is well known as the double-body linearization. The double-body surface elevation $\zeta_{b}$ is defined by:

$$
\zeta_{b}=-\frac{1}{2 g}\left(\nabla \phi_{d b} \cdot \nabla \phi_{d b}-2 U \frac{\partial \phi_{d b}}{\partial x}\right) \quad \text { at } z=\zeta_{b},
$$

which is a nonlinear equation and can be solved by iteration.

In the decomposition for $\bar{\phi}$, the time dependent part of the velocity potential $\phi$ is due to the oscillatory motion of the body in six degrees of freedom as:

$$
\phi=\sum_{k=1}^{6} \phi_{k} .
$$

\section{$2.2 \quad$ Free-surface boundary conditions}

From equation (2.4), the velocity potential is inserted into the nonlinear free-surface boundary conditions, and the resulting first-order terms are collected. Still one more nonlinearity is left, as the boundary conditions are evaluated at the surface elevation which is itself part of the solution, and is unknown a priori. Using Taylor series expansion, the free surface conditions can be instead evaluated at $z=0$. The results for the linearized dynamic and kinematic free-surface conditions are:

$$
\begin{array}{ll}
\frac{\partial \phi}{\partial t}=-g \zeta+U \frac{\partial \phi}{\partial x}-\nabla \phi_{b}^{\prime} \cdot \nabla \phi & \text { at } z=0 \\
\frac{\partial \zeta}{\partial t}=\frac{\partial \phi}{\partial z}+U \frac{\partial \zeta}{\partial x}-\nabla \phi_{b}^{\prime} \cdot \nabla \zeta+\zeta \frac{\partial^{2} \phi_{b}^{\prime}}{\partial z^{2}} & \text { at } z=0
\end{array}
$$

The base-flow potential $\phi_{b}^{\prime}$ is just zero in the case of the Neumann-Kelvin linearization, whereas for the double-body base flow, $\phi_{b}^{\prime}=\phi_{d b}$. We note that while $\phi_{b}$ here is taken to be $O(1)$, the steady wave 
elevation $\zeta_{b}$ has been assumed to be of $O(\epsilon)$, as argued by for example [6]. This allows the conditions to be applied on $z=0$, and terms arising from a Taylor expansion from $z=0$ to $z=\zeta_{b}$ are dropped.

Specifically, these terms are:

$$
\zeta \frac{\partial}{\partial z}\left(\nabla \zeta_{b} \cdot \nabla \phi_{b}\right) \quad \text { and } \quad \nabla \zeta_{b} \cdot \nabla \phi
$$

Note that the Neumann-Kelvin free-surface conditions can also be obtained when $\phi_{b}^{\prime}=0$ and $\zeta_{b}=0$ as follows:

$$
\begin{array}{ll}
\frac{\partial \phi}{\partial t}=-g \zeta+U \frac{\partial \phi}{\partial x} & \text { at } z=0 \\
\frac{\partial \zeta}{\partial t}=\frac{\partial \phi}{\partial z}+U \frac{\partial \zeta}{\partial x} & \text { at } z=0
\end{array}
$$

\subsection{Body boundary conditions}

Applying the body boundary condition for a floating body according to equation (A.9), requires that the exact instantaneous wetted surface of the body is known beforehand. This also adds to the nonlinearity of the formulation. This boundary condition can be linearized by first finding a firstorder relation between the body-fixed frame $\left(O^{\prime}-x^{\prime}, y^{\prime}, z^{\prime}\right)$ and the translating frame of reference $(O-x, y, z)$. Then with multiple use of Taylor series expansion, the nonlinear boundary condition can be linearized and evaluated at the mean position of the body surface $S_{0}$ which is known beforehand. This process is shown in detail in Appendix B, where a generalized linear body boundary condition is derived. This general boundary condition is valid for both rigid- and flexible-body modes of motion. In this paper we only present results for the rigid-body modes in which case the boundary condition can be written:

$$
\frac{\partial \phi_{k}}{\partial n}=\dot{\xi}_{k} \cdot n_{k}+\xi_{k} \cdot m_{k}, \quad \text { at } S_{0}
$$

In which:

$$
\begin{aligned}
\left(\xi_{1}, \xi_{2}, \xi_{3}\right) & =\left(\xi_{1}, \xi_{2}, \xi_{3}\right), \\
\left(\xi_{4}, \xi_{5}, \xi_{6}\right) & =\left(\Omega_{1}, \Omega_{2}, \Omega_{3}\right), \\
\left(n_{1}, n_{2}, n_{3}\right) & =\boldsymbol{n}, \\
\left(n_{4}, n_{5}, n_{6}\right) & =(\boldsymbol{r} \times \boldsymbol{n}), \\
\left(m_{1}, m_{2}, m_{3}\right) & =(\boldsymbol{n} \cdot \nabla)\left(\boldsymbol{W}-\nabla \phi_{b}^{\prime}\right), \\
\left(m_{4}, m_{5}, m_{6}\right) & =(\boldsymbol{n} \cdot \nabla)\left(\boldsymbol{r} \times\left(\boldsymbol{W}-\nabla \phi_{b}^{\prime}\right)\right) .
\end{aligned}
$$

where $\boldsymbol{r}$ is the position vector of a point on $S_{0}$.

\subsection{Bed and the far-field boundary conditions}

At the sea bed $S_{d}$ and the far-field truncation boundary $S_{\infty}$ the no-flux condition is applied as follows:

$$
\mathbf{n} \cdot \nabla \phi=0 .
$$

Note that ideally it is best to apply a suitable radiation boundary condition at $S_{\infty}$ which ensures all the far-field waves are outgoing from the domain. In this solver instead a very strong grid stretching is used so that virtually no waves are resolved toward the far-field boundary. In addition, the frequencycontent of the pseudo-impulsive body motion and the domain size are tuned such that no waves have time to reflect from the boundary and return to the ship before the simulation is completed. The above-mentioned boundary condition at $S_{\infty}$ is applied just to completely define a well-posed boundary value problem for the numerical calculations. 


\subsection{First-order forces}

The linearized solution for the velocity potential can be used to calculate the pressure and the resulting forces on the body. If the solution decomposition from (2.4) is applied also to the Bernoulli equation (A.2), then the resulting zeroth- and first-order pressure will be:

$$
\begin{aligned}
& p^{0}=-\rho\left\{\frac{1}{2}\left(\nabla \phi_{b}^{\prime}\right)^{2}-\boldsymbol{W} \cdot \nabla \phi_{b}^{\prime}\right\}, \\
& p^{1}=-\rho\left\{\left(\frac{\partial}{\partial t}-\boldsymbol{W} \cdot \nabla\right) \phi+\nabla \phi_{b}^{\prime} \cdot \nabla \phi\right\} .
\end{aligned}
$$

Integrating this first-order pressure over the surface of the body gives the resulting first-order forces which will be used to calculate the hydrodynamic coefficients for the radiation problem.

\section{The numerical method}

A numerical approximation of the solution to the linearized initial boundary value problem is developed. The above posed problem needs to be discretized both in time and space. A method of lines
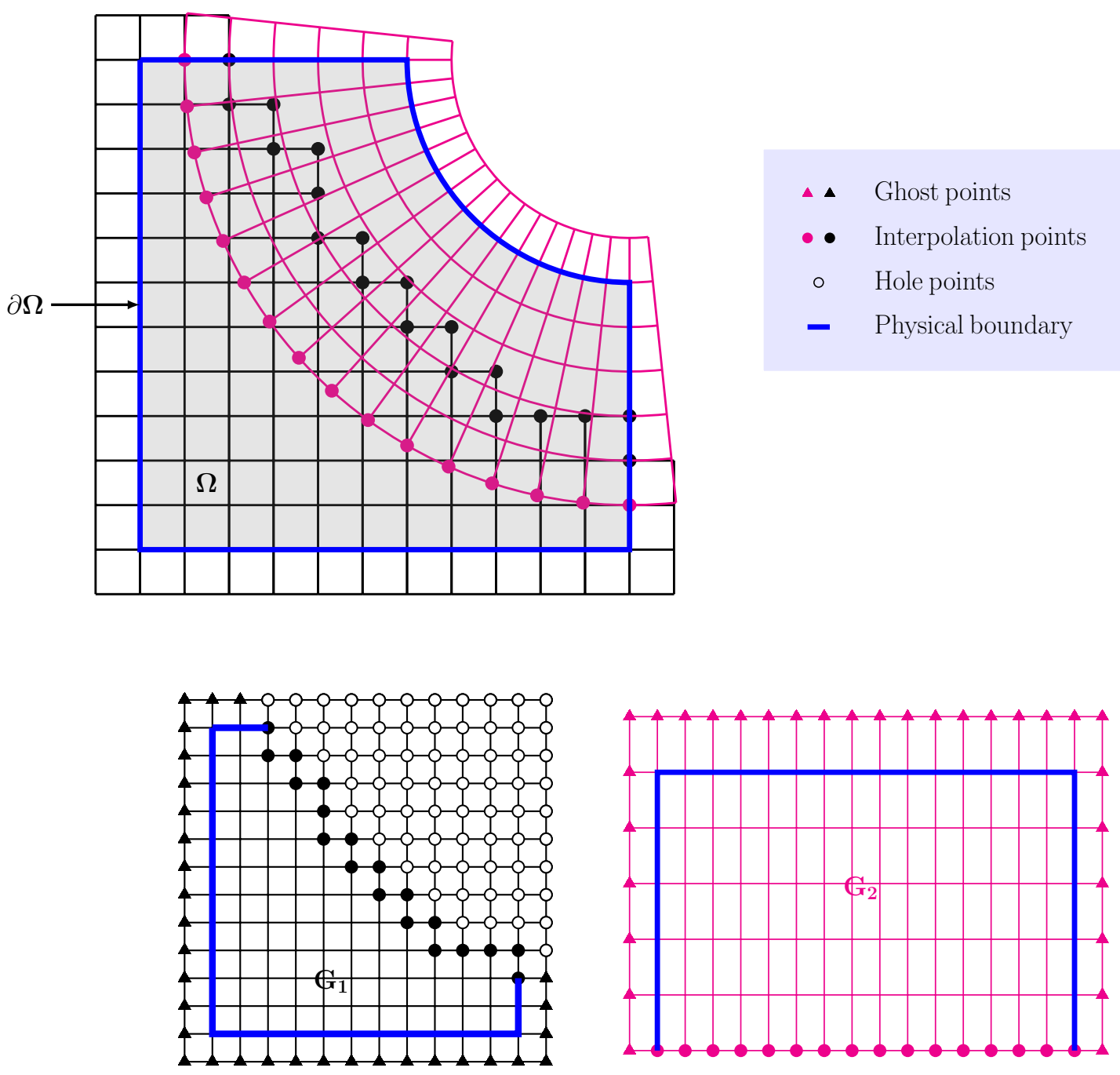

Figure 2: The overlapping grids in physical domain (top) and computational domain (bottom) 
approach is used here [19], where at each time step, first the continuity equation (2.3) is solved in the spatially discretized domain subjected to the relevant boundary conditions at the free surface, the body, the bed and the far-field boundary. From this solution, the right hand sides of the dynamic and the kinematic free-surface conditions, (2.11) and (2.12), can be computed. These equations can be used to compute the time rate of change of $\phi$ and and $\zeta$ which can be evolved forward in time using a suitable integration scheme.

\subsection{Spatial discretization}

For the spatial discretization, the 4th order finite-difference scheme is employed. This has been implemented inside Overture [20], which is an open-source collection of $\mathrm{C}++$ libraries for solving partial differential equations on overlapping grids using the finite-difference method. Using the mesh generator Ogen [21], an overlapping grid can be made for the spatial domain. A schematic drawing of an overlapping grid comprised of 2 component grids labelled G1 and G2 for a second-order discretization is shown in Figure 2. Grids are shown both in the physical domain and the computational domain where the finite-difference computation is carried out. For closed-form shapes like a cylinder or sphere, the transformation between the domains can be obtained analytically. For the more general grids like those for a ship hull, a numerical transformation will be applied. The set of G1 and G2 covers the entire computational domain $\boldsymbol{\Omega}$ including the body surface, which in this figure can be represented by the upper physical boundary of grid G2. Each component grid is comprised of the discretization points, the interpolation points, the ghost points and the hole points. The overlapping region in the grid is characterized by the interpolation points which are shown by the filled circles in Figure 2 . The solution at these points can be obtained by an interpolation from the discretization points or the interpolation points of the other component grids. Outside the domain boundaries, one or two layers of ghost points are generated for 2nd- and 4th-order schemes respectively, to allow for the use of centered stencils everywhere. In the figure one layer of the ghost points are shown by the filled triangles. The hole points, illustrated here by the open circles, do not influence the solution and are ignored.

\subsubsection{The system matrix}

The physical boundaries of the component grids which are connected to the surface of the body, will have a non-homogeneous Neumann-type boundary condition for $\phi$, as expressed by (2.15). For the physical boundaries representing the sea bed and the far-field boundary, homogeneous Neumann-type conditions are defined. At the physical boundaries representing the free surface, a Dirichlet-type boundary condition is applied to define the velocity potential $\phi$ at that location. At the discretization points the Laplace equation is applied. The relevant interpolation equation is also used for the interpolation points in the overlapping region. At Neumann boundaries, the first ghost layer is used to impose the Neumann condition, while the values at the second layer are obtained by extrapolation. At Dirichlet boundaries, the values of both layers of ghost points are obtained by extrapolation.

This set of discretized equations is finally assembled into the coefficient matrix $[\boldsymbol{A}]$ to produce the linear system of equations:

$$
[\boldsymbol{A}] \phi=[b],
$$

where $b$ is the vector comprised of zeros for the points with the Laplace equation, and the relevant forcing for the points with non-homogeneous boundary conditions or interpolation/extrapolation conditions. The $[A]$ sparse matrix is set as follows [22]:

- First the matrix is populated by the coefficients for 4th order discretization of the Laplace operator at all interior and the boundary grid points.

- Next at the the Dirichlet boundary, the coefficients are replaced with those related to the identity operator. 
- Points at the first ghost layer next to the Dirichlet boundaries are populated by the extrapolation coefficients.

- Points at the first ghost layer next to the Neumann boundaries are populated using the Neumann operator.

- Finally at all remaining ghost points and the corner points, the extrapolation coefficients are applied.

\subsubsection{Interpolation between component grids}

The solution in the overset regions is obtained via interpolation between the grids. To illustrate the procedure, consider two component grids (in two-dimensions) with corresponding solutions $\phi_{1}(i, j)$ and $\phi_{2}(i, j)$. The interpolation conditions can be written as follows [23]:

$$
\begin{aligned}
\phi_{1}(i, j) & =\sum_{k, l} \alpha_{1}(i, j, k, l) \phi_{2}(k, l), \\
\phi_{2}(i, j) & =\sum_{k, l} \alpha_{2}(i, j, k, l) \phi_{1}(k, l),
\end{aligned}
$$

where the interpolation coefficients are denoted by $\alpha$. The solution at the indices $i$ and $j$ is obtained by interpolating the solution at indices $k$ and $l$ from the other component grid. Both the explicit and implicit interpolation scheme can be employed. In the explicit interpolation, all values on the right hand side of (3.2) and (3.3) are the discretization points, whereas in the implicit interpolation some or all points from the right hand side of the equations are themselves interpolation points. This requires a system of equation to be solved to find the interpolated values. In the implicit case, the interpolation equations are included in the system matrix $[\boldsymbol{A}]$.

The interpolation schemes are a standard part of the Overture library. More details can be found in [23] and [20], where it is also shown how the width of the overlapping region and the order of interpolation scheme is chosen in a such a way that the overall order of accuracy of the scheme is always preserved.

\subsection{Temporal discretization}

The initial conditions provide the starting values of the surface elevation $\zeta$ and the velocity potential $\phi$ on the free-surface. The time rate of change of $\zeta$ and $\phi$ at the free surface are computed as described above, and they are evolved forward in time using the classical explicit 4th-order, 4-stage Runge-Kutta scheme. This process is then repeated until the end of the simulation is reached.

\subsubsection{Treatment of the convective derivatives}

Special care is required to ensure stability when working in a moving frame of reference. From the freesurface boundary conditions (2.11) and (2.12), we can identify four terms which represent convection:

$$
U \frac{\partial \phi}{\partial x}, \quad U \frac{\partial \zeta}{\partial x}, \frac{\partial \phi_{b}^{\prime}}{\partial y} \frac{\partial \phi}{\partial y}, \frac{\partial \phi_{b}^{\prime}}{\partial y} \frac{\partial \zeta}{\partial y} .
$$

These convective derivatives in the computational domain can be calculated as:

$$
\begin{aligned}
& \frac{\partial u}{\partial x}=\frac{\partial r}{\partial x} \frac{\partial u}{\partial r}+\frac{\partial s}{\partial x} \frac{\partial u}{\partial s}, \\
& \frac{\partial u}{\partial y}=\frac{\partial r}{\partial y} \frac{\partial u}{\partial r}+\frac{\partial s}{\partial y} \frac{\partial u}{\partial s} .
\end{aligned}
$$



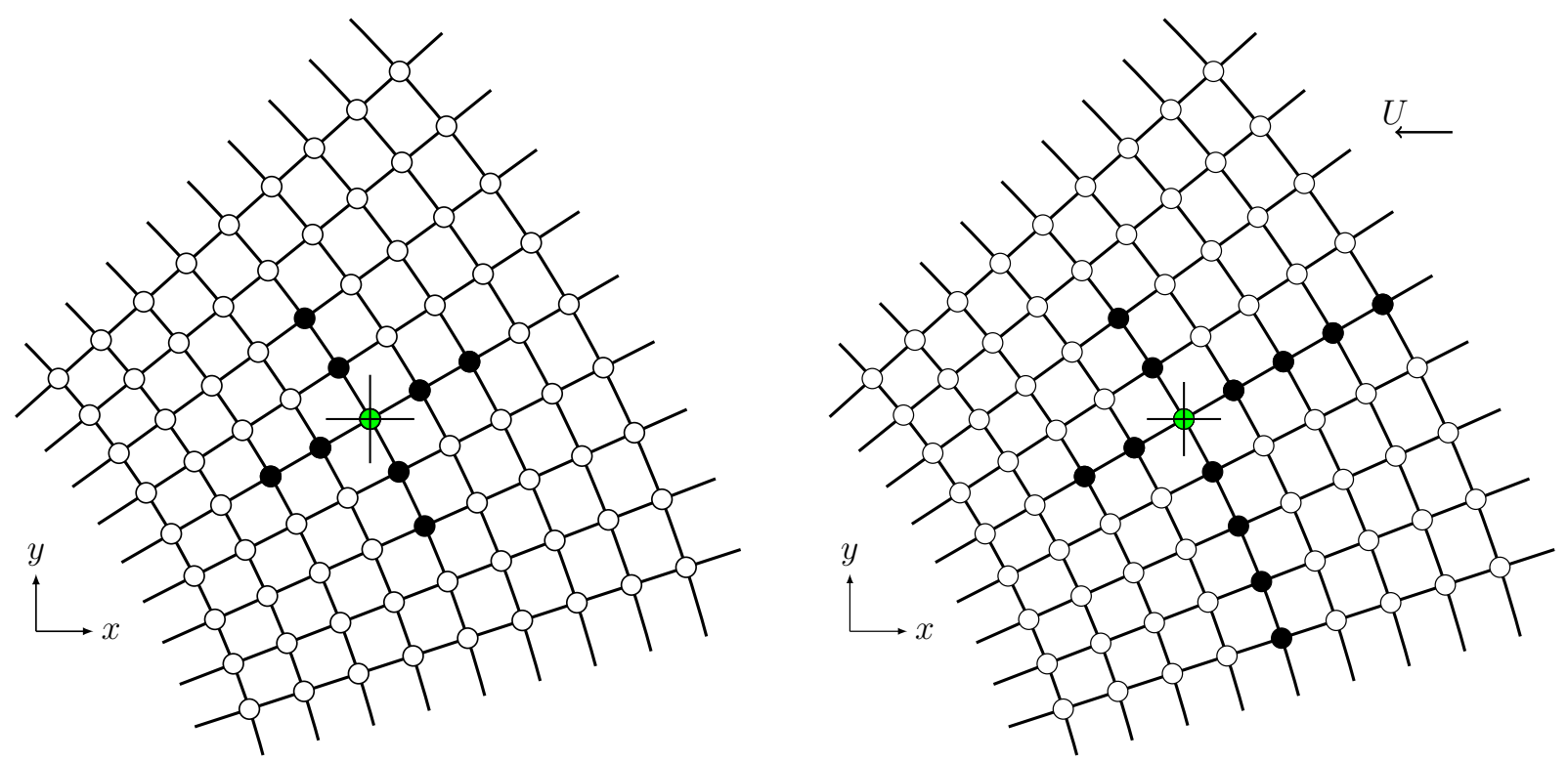

Figure 3: The centered 4th-order stencil (left) and the upwind 6th-order stencil (right) for $x$ convective derivatives

where the metrics of the transformation are denoted by $\partial r / \partial x, \partial s / \partial x, \partial r / \partial y$, and $\partial s / \partial y$. Here $u$ represents either the velocity potential $\phi$ or the surface elevation $\zeta$. To ensure the stability of the numerical scheme, an upwind biased stencil is employed to compute the derivatives of $u$ with respect to the unit spaced, Cartesian computational grid, $(r, s)$. For the convective $x$ derivatives, first the upwind direction in the physical domain is specified as the direction along the grid line where the potential $U x$ is increasing. This situation is illustrated in Figure 3, where the convective $x$ derivative is desired for the point marked with the cross. The left-hand plot here shows the original $4^{\text {th }}$-order, 5 point centered computational stencil, while the right-hand plot shows the upwinded $6^{\text {th }}$-order accurate, 7-point stencil. This scheme has the following coefficients:

$$
c_{i-2}=\frac{1}{30}, \quad c_{i-1}=-\frac{2}{5}, \quad c_{i}=-\frac{7}{12}, \quad c_{i+1}=\frac{4}{3}, \quad c_{i+2}=-\frac{1}{2}, \quad c_{i+3}=\frac{2}{15}, \quad c_{i+4}=-\frac{1}{60},
$$

where $i$ is the index along the $r$ or $s$ direction in the mapped domain. We have chosen to apply a $6^{\text {th }}$-order scheme, in order to offset the larger truncation errors associated with upwind differencing vs. central differencing.

For the $y$ convective derivatives, a local basis flow $y$-velocity is defined as:

$$
u_{l}=\frac{\partial \phi_{b}^{\prime}}{\partial y}
$$

using central differencing. Similar to the $x$ upwind derivatives, the upwind direction is determined by the direction along which $u_{l} y$ is increasing. Then the above-mentioned procedure for calculation of the $x$ convective derivatives is applied to the $y$ convective derivatives. Note that through this approach there are two separate biased stencils for the $x$ and $y$ upwind derivatives respectively.

\subsubsection{Treatment of the ghost points at the boundaries}

At the boundaries where it is required to set the ghost points before computing the convective derivatives two different conditions are used. This is shown in Figure 4, where inflow and outflow regions are defined as:

$$
\begin{array}{ll}
\boldsymbol{n} \cdot \boldsymbol{W}<0 & \text { the outflow region, } \\
\boldsymbol{n} \cdot \boldsymbol{W}>0 & \text { the inflow region. }
\end{array}
$$




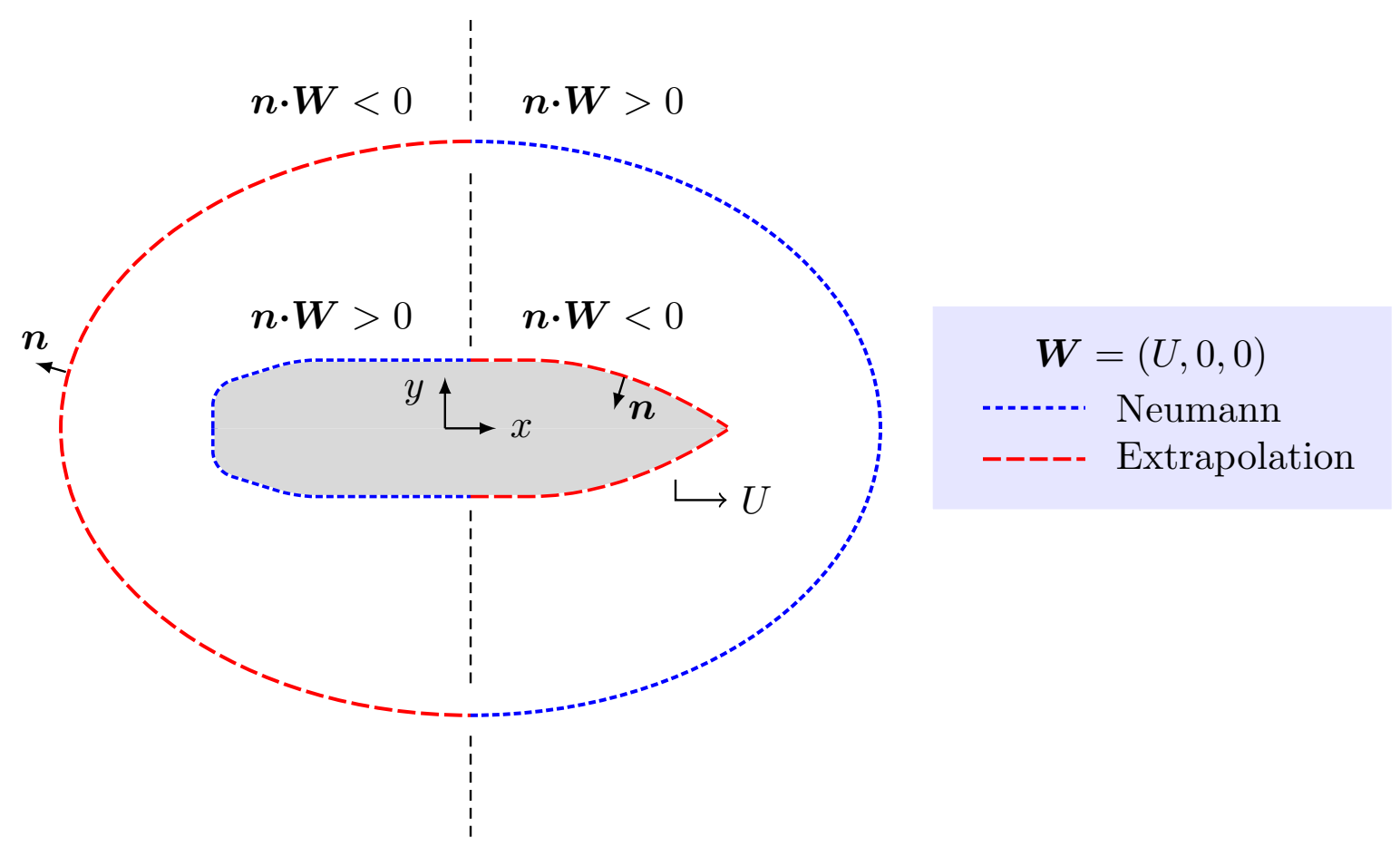

Figure 4: Definition of the inflow and outflow regions.

Extrapolation is used at inflow boundaries, while at outflow boundaries we impose a Neumann condition to obtain the ghost point value. The Neumann condition for the velocity potential $\phi$ is simply the body boundary condition (3.1). For the surface elevation $\zeta$, and in the case of the Neumann-Kelvin linearization, a new condition can be derived by taking the normal derivative of (2.14) which leads to:

$$
\frac{\partial \zeta}{\partial n}=-\frac{1}{g}\left(\frac{\partial}{\partial t}-U \frac{\partial}{\partial x}\right) \frac{\partial \phi}{\partial n} .
$$

A similar condition can also be obtained for the double-body linearization. It is important to mention that almost the same results have been obtained when a homogeneous Neumann condition has been employed instead of the inhomogeneous form given by (3.8), and this has been applied here for simplicity.

\subsubsection{Convergence studies}

In this section we verify the 4th-order numerical accuracy of the scheme using two simple problems with closed-form solutions. Figure 5 plots the maximum error in the numerical solution for the doublebody flow velocity potential on a floating sphere, compared to the analytical solution for a travelling dipole [24] given by:

$$
\phi_{d b}=-\left(\frac{1}{2} U a^{3} / r^{2}\right) \cos \theta .
$$

Here the radius of the sphere is $a$, the flow velocity is $U$ and $(r, \theta)$ are polar coordinates in the bodyfixed frame. The data is plotted on a $\log -\log$ scale, and the 1 to 4 slope line is also shown, indicating the expected rate of convergence.

Next we consider a pure wave propagation problem on a two-dimensional, periodic domain. The exact linear wave solution:

$$
\begin{aligned}
\phi_{0} & =\operatorname{Re}\left(\frac{\mathrm{i} g A}{\omega} e^{-\mathrm{i} k x}\right), \\
\zeta_{0} & =\operatorname{Re}\left(A e^{-\mathrm{i} k x}\right),
\end{aligned}
$$




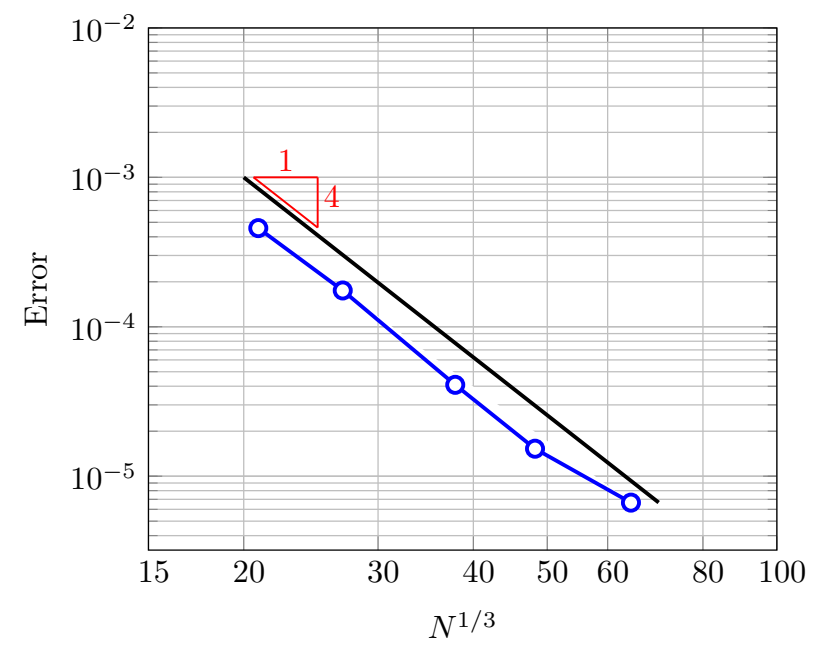

Figure 5: Convergence of the solution to the three-dimensional traveling dipole (double body for a sphere). The maximum error on the velocity potential around the sphere is plotted versus the grid spacing. $N$ denotes the number of grid points.

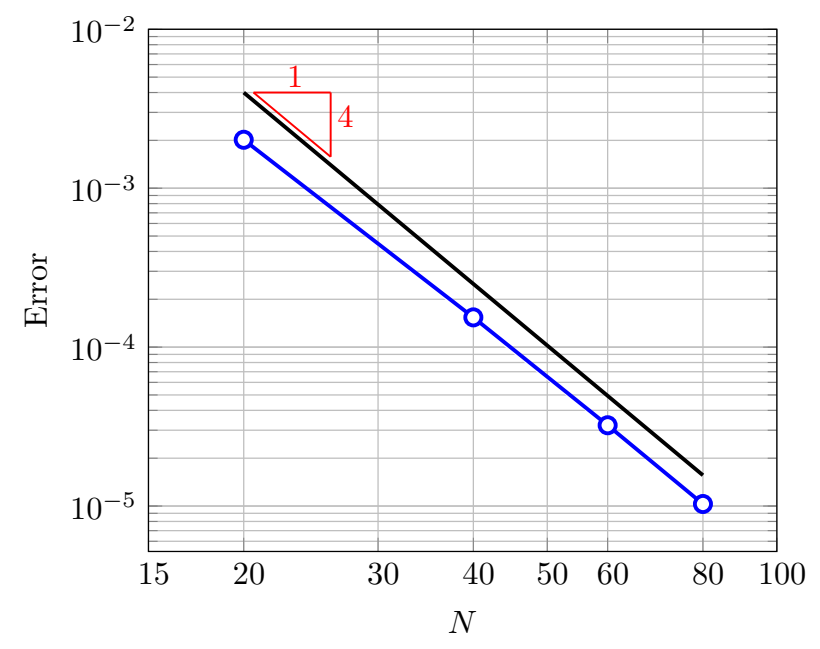

Figure 6: Convergence of the solution to the two-dimensional linear monochromatic wave. The error on the surface elevation per wave period is plotted versus the number of grid points $N$ at the free surface.

defines the initial conditions, and the code is run for 5 wave periods. Here $A$ denotes the wave amplitude, $\omega$ the wave frequency, and $k=2 \pi / \lambda$ the wave number for a linear wave with the length $\lambda$. The maximum error in the solution per wave period of propagation vs. the number of horizontal grid points is plotted on a log-log scale in Figure 6. Here the Neumann-Kelvin linearization has been applied with a velocity of $U=0.4 C_{p}$, where $C_{p}$ denotes the wave phase speed. Again, we see the expected 4th-order convergence of the method.

While all the numerical approximations applied in this solver are formally at least fourth-order accurate, we may not always be able to achieve such convergence in practice for more complicated problems due to several factors including: the finite depth of the computational domain, the truncated frequency-content of the pseudo-impulsive forcing, the asymptotic continuation applied to the Fourier transform, and for ship-like geometries, grid quality issues near areas of large curvature. We also note that since there are only two grid lines outside the solid domain boundaries at the free-surface, the upwind biased convection schemes at a small number of points near up-stream boundaries is reduced to second order accuracy. 


\subsection{Computational time}

As mentioned above, the coefficient matrix in this solver is sparse, and it is expected that the computational time will increase linearly with increasing number of grid points for an optimal iterative solution. To demonstrate this, an overset grid is prepared for a submerged sphere with increasing resolution. The time-domain calculation is conducted for the heave mode to get the force impulse. For this case the Generalized Minimal Residual iterative solver together with the incomplete LU Preconditioner has been used to solve the system of equations. The computational time is plotted in Figure 7 on a $\log -\log$ scale. As the number of grid points increases, the computational time approaches a linear scaling as expected.

\section{Pseudo-impulsive time-domain solution}

The design of the pseudo-impulsive motion for the numerical solution in the time-domain is described in this section. For each computational grid, an average grid spacing $l_{a}$ around the waterline or over the free-surface (for submerged bodies) is obtained. It is assumed that the minimum resolvable wavelength in the simulation is twice the average grid spacing. This is based on the spatial Nyquist wavenumber which can be defined by $k_{n}=\pi / l_{a}$ that is in fact half of the spatial sampling rate. Accordingly the maximum resolvable cyclic encounter frequency $f_{\max }$ can be calculated using the dispersion relation as follows:

$$
f_{\max }=\frac{1}{2 \pi}\left|\sqrt{g k_{n} \tanh \left(k_{n} h\right)}-k_{n} U\right|,
$$

where $h$ is the depth of the computational domain. In principle, it would be possible to capture frequencies from 0 to $f_{\max }$, but in practice due to the finite computational domain size, we also limit the lowest resolvable frequency to be that which corresponds to a wavelength equal to the domain radius. Thus based on the size of the computational domain and the average grid spacing, the minimum and maximum resolvable frequencies can be calculated. The time domain signal is also zero padded so as to the frequency resolution $d f$ is well below the lowest resolvable frequency.

The Fourier transform pair for a function $\phi$, based on the angular encounter frequency $\omega_{e}=2 \pi f$

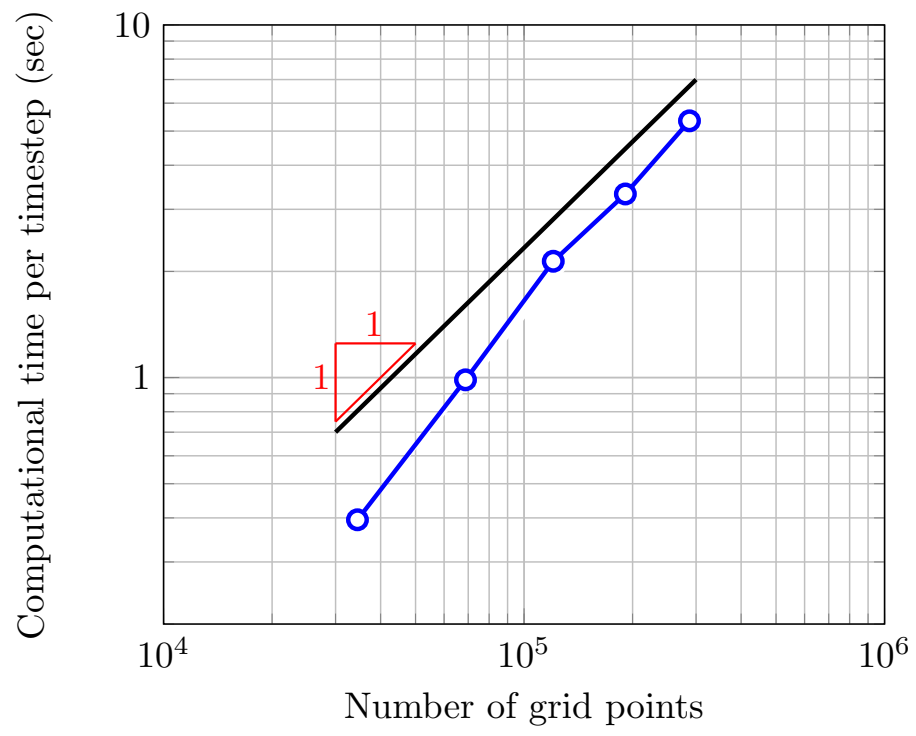

Figure 7: Scalability of the computation. The computation is related to a submerged sphere heaving with the Froude number $F n=0.4$. The iterative solver with incomplete LU Preconditioner is used. 
can be defined by:

$$
\begin{gathered}
\mathcal{F}\{\phi(t)\}=\hat{\phi}\left(\omega_{e}\right)=\int_{-\infty}^{\infty} \phi(t) e^{-i \omega_{e} t} d t, \\
\mathcal{F}^{-1}\left\{\hat{\phi}\left(\omega_{e}\right)\right\}=\phi(t)=\frac{1}{2 \pi} \int_{-\infty}^{\infty} \hat{\phi}\left(\omega_{e}\right) e^{i \omega_{e} t} d \omega_{e} .
\end{gathered}
$$

In this solver, instead of the impulsive body boundary condition, a pseudo-impulsive Gaussian relation is considered for the displacement of the body $\xi_{k}$ in the $k$ th direction as follows:

$$
\xi_{k}(t)=e^{-2 \pi^{2} s^{2} t^{2}}
$$

where $s$ is a parameter to control the variance or the range of the desired frequencies in the body motion. The velocity and the displacement of the body at each time step in equation (2.15) can be obtained from this equation. According to the above-mentioned Fourier transforms, the displacement of the body from equation (4.4), in the frequency domain would be:

$$
\hat{\xi}_{k}(f)=\frac{1}{s \sqrt{2 \pi}} e^{-f^{2} / 2 s^{2}}
$$

If $r$ is defined as $\hat{\xi}_{k}\left(f_{\max }\right) / \hat{\xi}_{k}(0)$, then the parameter $s$ will be:

$$
s=\sqrt{\frac{-f_{\max }^{2}}{2 \ln (r)}} .
$$

After choosing a suitable value for $r$, the shape of the pseudo-impulsive boundary condition can be obtained. Moreover the time-domain pseudo-impulse equation from (4.4) is shifted in time as follows:

$$
\xi_{k}(t)=e^{-2 \pi^{2} s^{2}\left(t-t_{0}\right)^{2}},
$$

in which $t_{0}=4 /(\pi s)$. This is to ensure that the displacement of the body at the start of simulation is zero. The amount of the shift is determined such that the following is satisfied:

$$
\bar{t}=t \sqrt{g / L} \approx 15,
$$

where $L$ is a characteristic length of the body. This is selected to follow the practice from the TIMIT time-domain solver [25], where the simulation time is selected so the non-dimensional time scale $\bar{t}$ is at least 15. Physically this means that there would be enough information captured in the time-domain signals of the simulation to obtain a reasonable Fourier transform. To ensure sufficient accuracy and stability of the numerical scheme, the simulation time step is chosen to be:

$$
\Delta t=\frac{C r \Delta x_{\min }}{u_{\max }}
$$

where the Courant number is chosen in the range of $0.5<C r<1$, and the maximum possible wave velocity is $u_{\max }=\sqrt{g h}$. The minimum grid spacing over the whole free surface is denoted here by $\Delta x_{\text {min }}$. After the computational set-up has been introduced, it is shown in the following section how the hydrodynamic coefficients can be calculated from the force time signal. 


\section{Frequency-domain transformation}

First consider the relation between the time- and frequency-domain formulations. The hydrodynamic forces in the forward speed radiation problem can be expressed as follows, see for example [5], [15]:

$$
\begin{aligned}
a_{j k}\left(\omega_{e}\right) & =a_{j k}^{\infty}-\frac{c_{j k}^{\infty}+c_{j k}^{0}}{\omega_{e}^{2}}-\frac{1}{\omega_{e}^{2}} \operatorname{Re}\left\{\int_{0}^{\infty} K_{j k}(t) e^{-i \omega_{e} t} d t\right\}, \\
b_{j k}\left(\omega_{e}\right) & =b_{j k}^{\infty}+\frac{1}{\omega_{e}} \operatorname{Im}\left\{\int_{0}^{\infty} K_{j k}(t) e^{-i \omega_{e} t} d t\right\}, \\
F_{j k}(t) & =-a_{j k}^{\infty} \ddot{x}(t)-b_{j k}^{\infty} \dot{x}(t)-\left(c_{j k}^{\infty}+c_{j k}^{0}\right) x(t)-\int_{-\infty}^{t} K_{j k}(t-\tau) x_{k}(\tau) d \tau .
\end{aligned}
$$

Here the radiation impulse response function is $K_{j k}$, and the convolution integral is defined based on the displacement of the body in mode $k$ given by $x_{k}$. Note that the forces which arise just during the impulsive body motion are proportional to $a_{j k}^{\infty}, b_{j k}^{\infty}, c_{j k}^{\infty}$ and $c_{j k}^{0}$. The first two are the infinitefrequency added mass and damping, and the last two are forward-speed hydrostatic coefficients. These quantities which are due to the so called pressure release problem can be obtained by solving the relevant boundary value problems which are given in [5]. The total hydrodynamic force $F_{j k}$ is then the force exerted on the body during and also after the impulsive displacement. The corresponding added mass and damping coefficients in the frequency domain are $a_{j k}\left(\omega_{e}\right), b_{j k}\left(\omega_{e}\right)$ respectively.

Using (5.1) and (5.2) it is easy to verify that:

$$
\begin{array}{r}
\operatorname{Re}\left\{\int_{0}^{\infty} K_{j k}(t) e^{-\mathrm{i} \omega_{e} t} d t\right\}+\mathrm{i} \operatorname{Im} \\
\left\{\int_{0}^{\infty} K_{j k}(t) e^{-\mathrm{i} \omega_{e} t} d t\right\}=\int_{0}^{\infty} K_{j k}(t) e^{-\mathrm{i} \omega_{e} t} d t=\mathcal{F}\left\{K_{j k}(t)\right\} \\
=-\omega_{e}^{2} a_{j k}\left(\omega_{e}\right)+\mathrm{i} \omega_{e} b_{j k}\left(\omega_{e}\right)+\omega_{e}^{2} a_{j k}^{\infty}-\mathrm{i} \omega_{e} b_{j k}^{\infty}-\left(c_{j k}^{\infty}+c_{j k}^{0}\right) .
\end{array}
$$

The convolution from (5.3) can be performed by multiplication of the the Fourier transforms in the frequency domain as:

$$
\int_{-\infty}^{t} K_{j k}(t-\tau) x_{k}(\tau) d \tau=\mathcal{F}^{-1}\left\{\mathcal{F}\left\{K_{j k}(t)\right\} \mathcal{F}\left\{x_{k}(t)\right\}\right\}
$$

Then by taking the Fourier transform of both sides of the equation (5.3) we arrive at:

$$
\begin{aligned}
\mathcal{F}\left\{K_{j k}(t)\right\} \mathcal{F}\left\{x_{k}(t)\right\}=-\mathcal{F}\left\{F_{j k}(t)+a_{j k}^{\infty} \ddot{x}(t)+b_{j k}^{\infty} \dot{x}(t)+\left(c_{j k}^{\infty}+c_{j k}^{0}\right) x(t)\right\}= \\
\quad-\mathcal{F}\left\{F_{j k}(t)\right\}+\left(\omega_{e}^{2} a_{j k}^{\infty}-i \omega_{e} b_{j k}^{\infty}-c_{j k}^{\infty}-c_{j k}^{0}\right) \mathcal{F}\left\{x_{k}(t)\right\} .
\end{aligned}
$$

If we insert $\mathcal{F}\left\{K_{j k}(t)\right\}$ from (5.4) then:

$$
\begin{array}{r}
{\left[-\omega_{e}^{2} a_{j k}\left(\omega_{e}\right)+\mathrm{i} \omega_{e} b_{j k}\left(\omega_{e}\right)+\omega_{e}^{2} a_{j k}^{\infty}-\mathrm{i} \omega_{e} b_{j k}^{\infty}-\left(c_{j k}^{\infty}+c_{j k}^{0}\right)\right] \mathcal{F}\left\{x_{k}(t)\right\}=} \\
-\mathcal{F}\left\{F_{j k}(t)\right\}+\left(\omega_{e}^{2} a_{j k}^{\infty}-\mathrm{i} \omega_{e} b_{j k}^{\infty}-c_{j k}^{\infty}-c_{j k}^{0}\right) \mathcal{F}\left\{x_{k}(t)\right\}
\end{array}
$$

Finally we see that:

$$
\omega_{e}^{2} a_{j k}-\mathrm{i} \omega_{e} b_{j k}=\frac{\mathcal{F}\left\{F_{j k}(t)\right\}}{\mathcal{F}\left\{x_{k}(t)\right\}} .
$$

For the computation of the added mass and damping coefficients it is required just to consider the Fourier transform of the force and the displacement time signals. Note that the frequency space for the discrete Fourier transform is defined based on the encounter frequency $\omega_{e}$. 


\subsection{The critical frequency when $\tau_{c}=U \omega_{c} / g=1 / 4$}

For completeness, we review here some of the subtleties associated with the forward-speed radiation problem in connection with the critical frequency at $\tau=1 / 4$. In contrast to the case of zero forward speed, an oscillating and moving body will generate more than one wave system. The significance of the non dimensional term $\tau=U \omega_{e} / g$ is also due to this fact. For two-dimensional bodies it is straitforward to obtain these wave systems. Assuming that the velocity potential $\hat{\phi}_{k}$ in this case satisfies the combined free-surface condition based on the Neumann-Kelvin linearization, then in the frequency domain:

$$
\frac{\partial \hat{\phi}_{k}}{\partial z}+\frac{\tau^{2}}{\nu} \frac{\partial^{2} \hat{\phi}_{k}}{\partial x^{2}}-2 \mathrm{i} \tau \frac{\partial \hat{\phi}_{k}}{\partial x}-\nu \hat{\phi}_{k}=0, \quad \text { at } z=0 .
$$

in which $\nu=\omega_{e}^{2} / g$. The velocity potential in time domain is defined by:

$$
\phi_{k}(t)=\operatorname{Re}\left\{\hat{\phi} e^{i \omega_{e} t}\right\}
$$

Let us consider the asymptotic solutions in the far-field:

$$
\begin{array}{ll}
\hat{\phi}_{k}^{-}=\frac{g}{\omega} A_{k}^{-} e^{-\mathrm{i} k x} e^{k z} & \text { as } x \rightarrow+\infty, \\
\hat{\phi}_{k}^{+}=\frac{g}{\omega} A_{k}^{+} e^{\mathrm{i} k x} e^{k z} & \text { as } x \rightarrow-\infty,
\end{array}
$$

where $k$ is the wave number and $\omega$ is the wave frequency. The complex amplitude of the outgoing waves are denoted by $A_{k}^{-}$and $A_{k}^{+}$, which are constant values due to the two-dimensionality of the problem. If the asymptotic solutions are inserted in to the combined condition then:

$$
\begin{array}{lll}
k^{2}-\frac{\nu}{\tau^{2}}(1-2 \tau) k+\frac{\nu^{2}}{\tau^{2}}=0 \quad \Rightarrow \quad k_{1,2}=\frac{g}{2 U^{2}}(1-2 \tau \pm \sqrt{1-4 \tau}) & \text { as } x \rightarrow+\infty, \\
k^{2}-\frac{\nu}{\tau^{2}}(1+2 \tau) k+\frac{\nu^{2}}{\tau^{2}}=0 \quad \Rightarrow \quad k_{3,4}=\frac{g}{2 U^{2}}(1+2 \tau \pm \sqrt{1+4 \tau}) & \text { as } x \rightarrow-\infty .
\end{array}
$$

With the same encounter frequency, there are 4 waves with different wavelengths. When $\tau<1 / 4$ the corresponding group velocities $C_{g}=1 / 2 \sqrt{g / k}$ can also be obtained as:

$$
\begin{aligned}
& C_{g}^{k_{1}}=U \frac{1}{\sqrt{2(1-2 \tau+\sqrt{1-4 \tau})},} 1<\sqrt{2(1-2 \tau+\sqrt{1-4 \tau})}<2 \Rightarrow C_{g}^{k_{1}}<U, \\
& C_{g}^{k_{2}}=U \frac{1}{\sqrt{2(1-2 \tau-\sqrt{1-4 \tau})}}, \quad 0<\sqrt{2(1-2 \tau-\sqrt{1-4 \tau})}<1 \Rightarrow C_{g}^{k_{2}}>U, \\
& C_{g}^{k_{3}}=U \frac{1}{\sqrt{2(1+2 \tau+\sqrt{1+4 \tau})}}, \quad \sqrt{2(1+2 \tau+\sqrt{1+4 \tau})}>2 \Rightarrow C_{g}^{k_{3}}<U, \\
& C_{g}^{k_{4}}=U \frac{1}{\sqrt{2(1+2 \tau-\sqrt{1+4 \tau})}}, \quad 0<\sqrt{2(1+2 \tau-\sqrt{1+4 \tau})}<1 \Rightarrow C_{g}^{k_{4}}>U .
\end{aligned}
$$

It is seen that in front of the body towards $+\infty$ only the $k_{2}$ wave has a group velocity larger than $U$. The $k_{1}$ wave system has a smaller group velocity than $U$, and it would be overtaken by the body. With respect to the moving frame it will propagate towards $-\infty$. Moreover the $k_{3}$ and $k_{4}$ waves propagate towards $-\infty$ regardless of their group velocity with respect to the body velocity. Thus if $\tau<1 / 4$, three waves are generated behind the body with the corresponding wavenumber $k_{1}, k_{3}$ and $k_{4}$, and only $k_{2}$ wave is generated in front of the body towards $+\infty$.

In the same manner, for the case of $\tau>1 / 4$, there would be no wave in front of the body as $k_{1}$ and $k_{2}$ become complex. Behind the moving body, two waves, i.e $k_{3}$ and $k_{4}$ would be generated 
propagating towards $-\infty$. The group velocity for the $k_{3}$ wave is less than the forward speed of the body $U$, whereas the group velocity for the $k_{4}$ wave is greater than $U$ only when $1 / 4<\tau<3 / 4$.

When $\tau=\tau_{c}=1 / 4$, the two wave systems $k_{1}$ and $k_{2}$ merge into a single wave with a wave number equal to $g / 4 U^{2}$. The corresponding group velocity is then equal to the forward speed of the body $U$. Additionally the $k_{3}$ and $k_{4}$ wave systems propagate towards $-\infty$ behind the body.

Using the Green function for an oscillating source located in a uniform current, a similar explanation regarding these wave systems is also given by [26]. Moreover a study of the wave systems in the case of three-dimensional problems can also be found in [27] and [28].

This illustrates how, at the critical frequency $\omega_{c}=g / 4 U$, part of the generated wave system is trapped (in $2 \mathrm{D}$, or nearly trapped in $3 \mathrm{D}$ ) to the moving body, as this energy propagates with the same speed as the moving body $U$. For impulsive or pseudo-impulsive time-domain simulations, some energy at this frequency will appear in the solution. This energy will radiate very slowly away from the body compared to all other frequencies, and thus take a very long time for the signal to die out. Instead of computing very long simulations however, we adopt the method suggested by [5] and [25], and assume an asymptotic behavior of the force signal of the form:

$$
F_{a}(t)=\frac{1}{t^{n}}\left[a_{1} \sin \left(\omega_{c} t\right)+a_{2} \cos \left(\omega_{c} t\right)\right] .
$$

The exponent $n$ determines the rate at which the oscillation with $\omega_{c}$ dies out. In order to find $a_{1}$ and $a_{2}$, the tail of the force signal is extrapolated in time using a least-squares fit to the above asymptotic relation for the force. To calculate the hydrodynamic coefficients based on (5.6), the Fourier transform of the asymptotic force $F_{a}(t)$, is combined with the Fourier transform of the force signal from the simulation. If the least-square fitting starts from time $t_{m}$ then the Fourier transform of the asymptotic contribution is

$$
\int_{t_{m}}^{t_{\infty}} \frac{1}{t}\left[a_{1} \sin \left(\omega_{c} t\right)+a_{2} \cos \left(\omega_{c} t\right)\right] e^{-\mathrm{i} \omega_{e} t} d t,
$$

where we have chosen a value of $n=1$ for the decay rate. This integral can be evaluated analytically and the solution is given by [5]. Some examples of the asymptotic least-square fitting to the radiation force signals are given in Figures 8 to 10. In the first case, the radiation force $F_{33}$ is plotted for a submerged two-dimensional cylinder. Next $F_{11}$ is given for a submerged sphere. Note that a close-up view of these forces are also presented in Figures 8 and 9 . Finally in Figure $10, F_{13}$ and $F_{31}$ are shown for the submerged sphere. Note also that according to [29], $F_{13}=-F_{31}$ as can be seen from the figure. For the more detailed specifications about these simulations refer to the next section. 

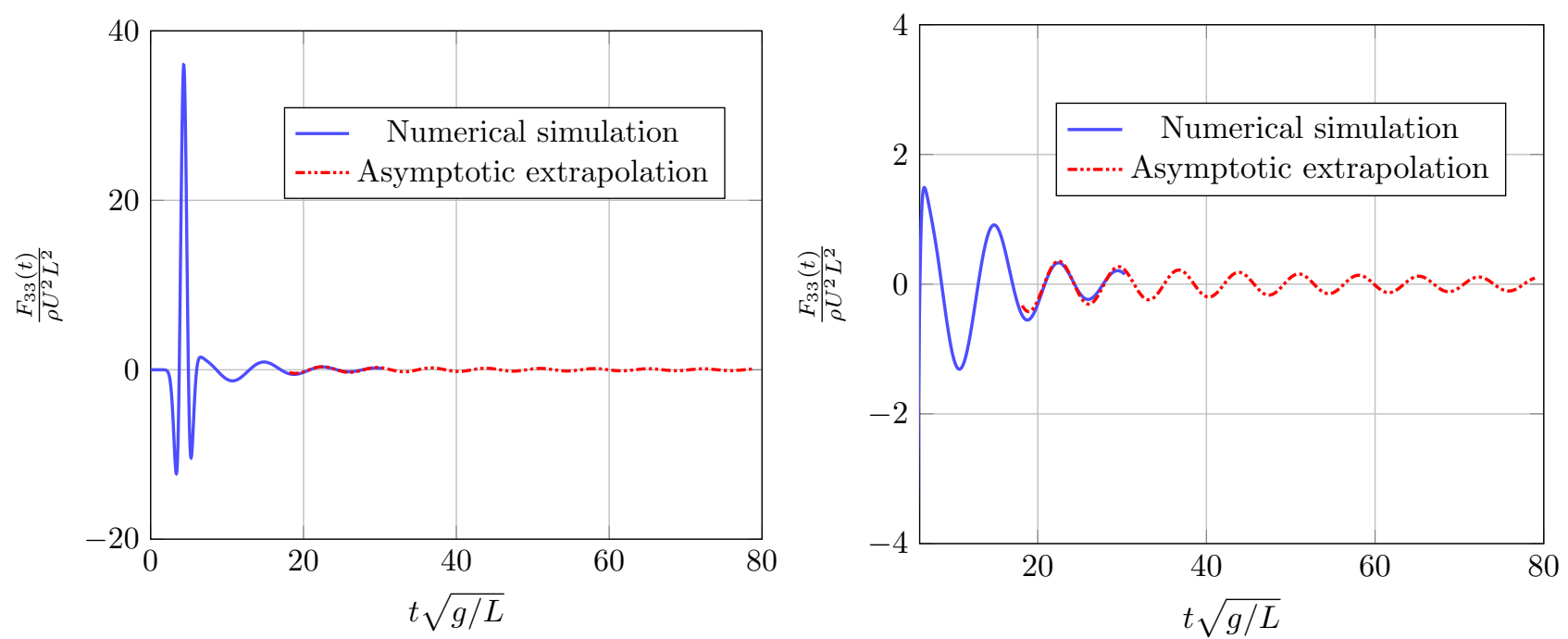

Figure 8: The asymptotic force oscillation with frequency $\omega_{c}$, submerged cylinder, $F_{33}$
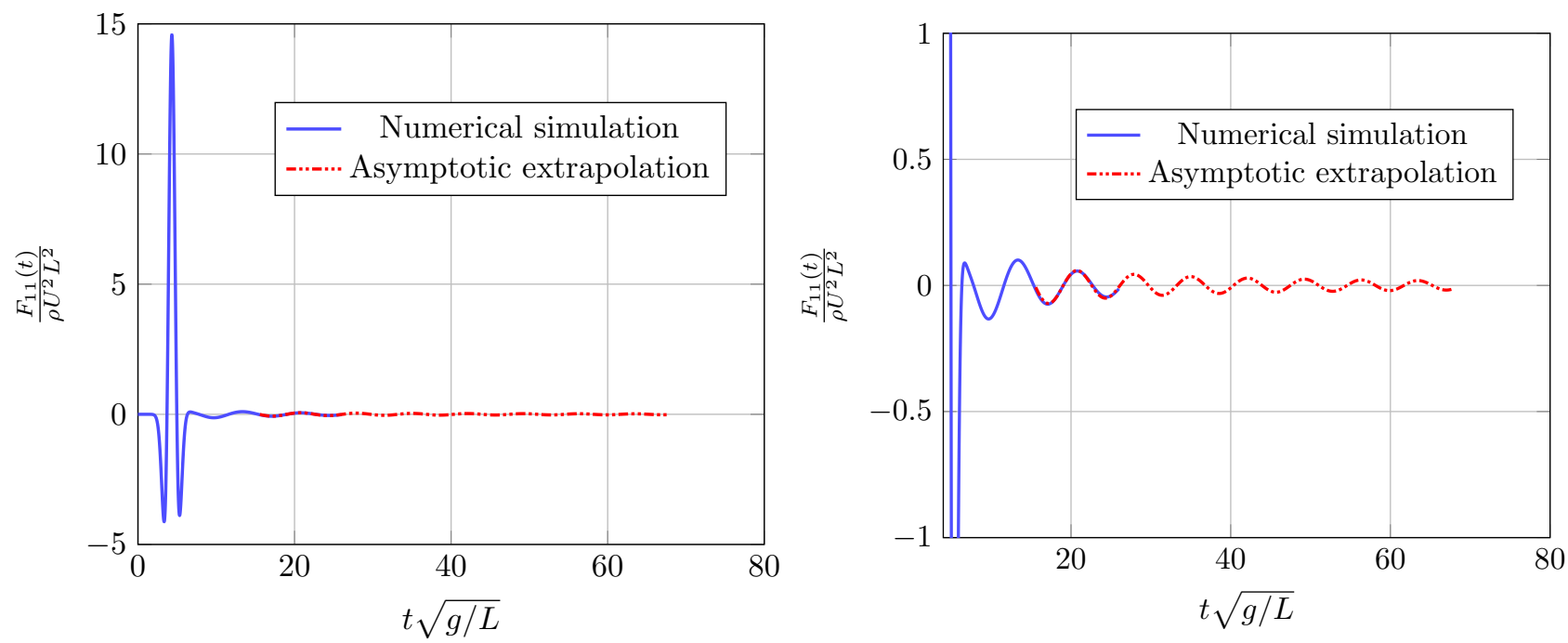

Figure 9: The asymptotic force oscillation with frequency $\omega_{c}$, submerged sphere, $F_{11}$
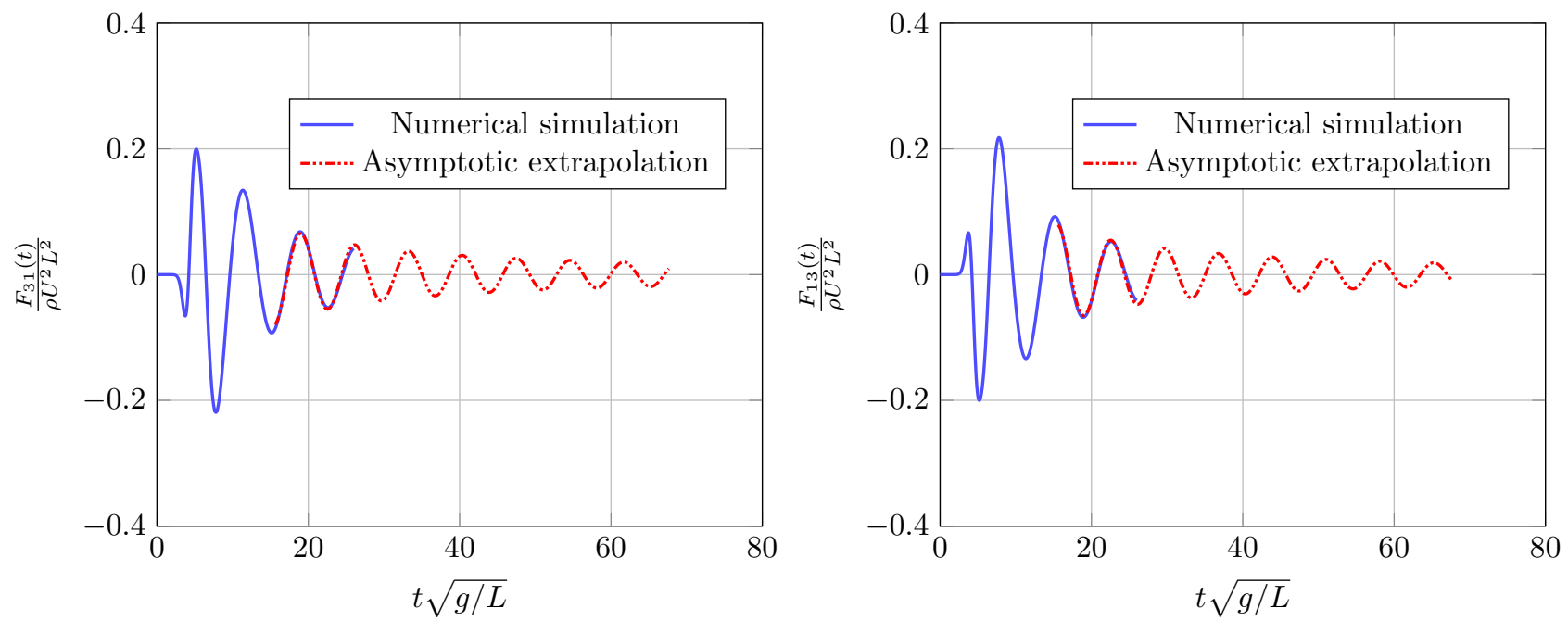

Figure 10: The asymptotic force oscillation with frequency $\omega_{c}$, submerged sphere, $\left(F_{31}\right.$ and $\left.F_{31}\right)$ 

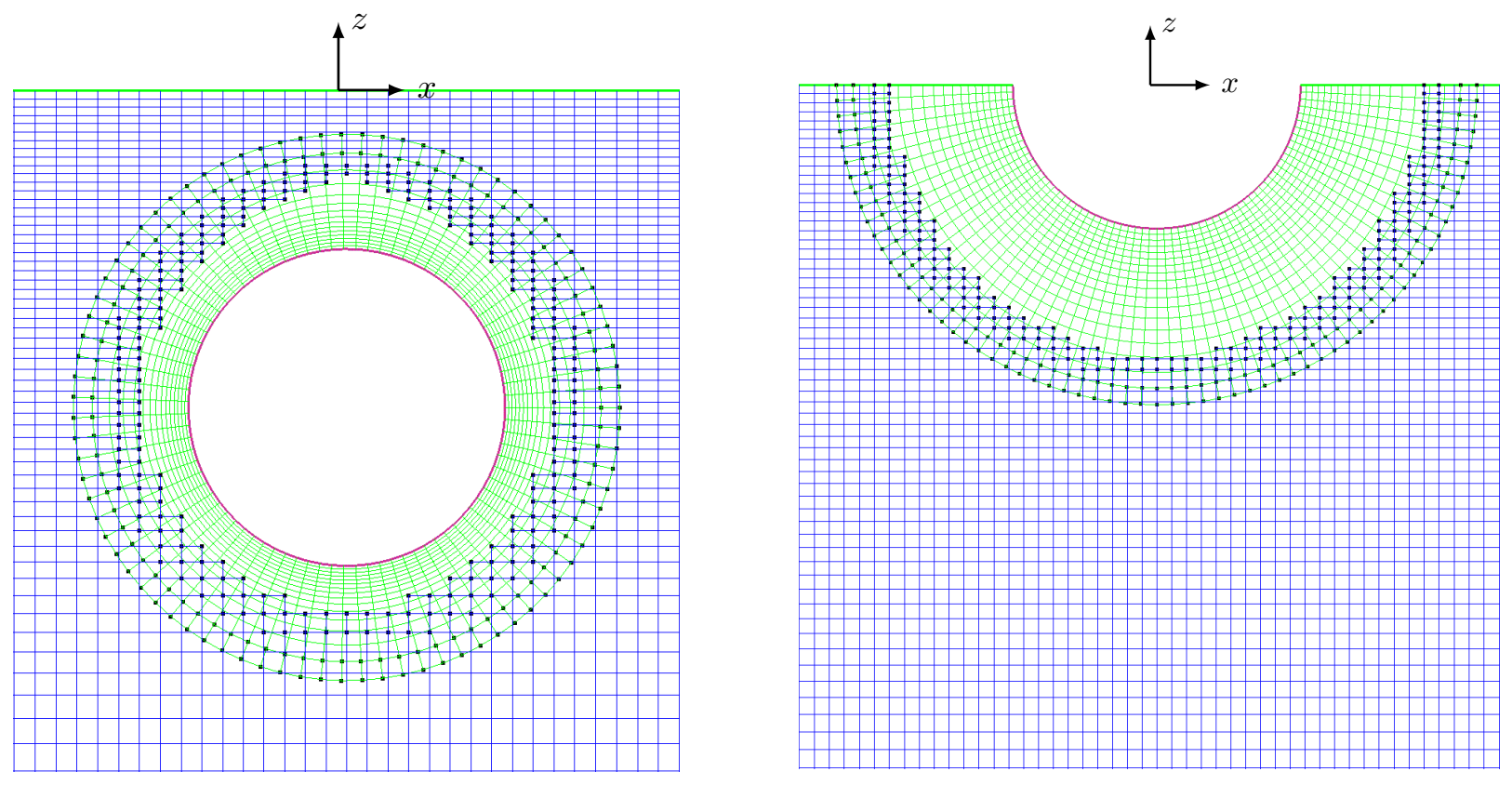

Figure 11: Part of the overlapping grid for the submerged and the floating cylinder

\section{Results}

The developed solver is used to calculate the hydrodynamic coefficients for some simple closed-form geometries with known solution and for one ship geometry. Note that the computational results obtained by the method presented here are given the label OceanWave3D-Seakeeping.

\subsection{Closed-form geometries}

For the closed form geometries (a cylinder and a sphere) the results are compared with the corresponding analytical or semi-analytical solutions given by [30], [31] and [32]. Note that these reference solutions are obtained assuming the Neumann-Kelvin free-surface condition (2.13) and (2.14). So, in order to solve exactly the same boundary-value problems as those which are posed in these references, the Neumann-Kelvin linearization is applied for the free-surface conditions. The frequency-domain equivalent of these conditions is also given in (5.7). The base flow $\phi_{b}^{\prime}$ for the body-boundary conditions in (2.15) for all computations including the closed-form geometries are of the double-body type $\phi_{d b}$, which is obtained from (2.5) to (2.8).

\section{The submerged cylinder (Low Froude number)}

For the first validation case, an overlapping grid is made for a two-dimensional submerged cylinder with radius $a$. See Figure 11. The body is forced to oscillate in surge and heave, and it moves with the Froude number $F r=U / \sqrt{g a}=0.03$ in the positive x direction. The submergence depth of the cylinder $d$ is equal to $2 a$. In Figure 12 the computed added mass and damping coefficients $a_{13}, b_{13}$ are compared with the analytical coefficients from [30].

\section{The floating cylinder (Low Froude number)}

The hydrodynamic coefficients for a floating two-dimensional cylinder are also calculated numerically. The overlapping grid is shown in Figure 11. The body has a Froude number of $F r=U / \sqrt{g a}=0.03$ in the positive $\mathrm{x}$ direction. The comparison is made with the analytical results obtained by [30], and the results are shown in Figure 13. 

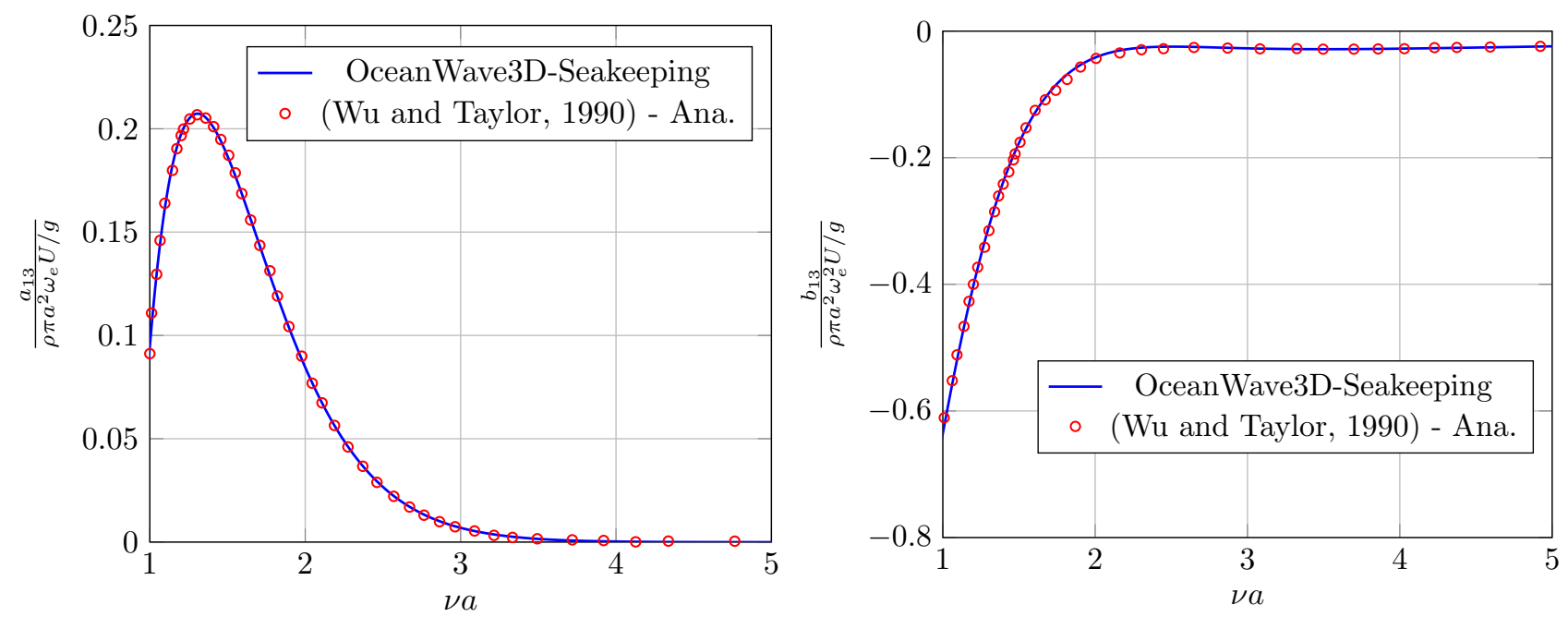

Figure 12: The hydrodynamic coefficients $\left(a_{13}\right.$ and $\left.b_{13}\right)$ for the submerged cylinder, $\mathrm{Fr}=0.03$
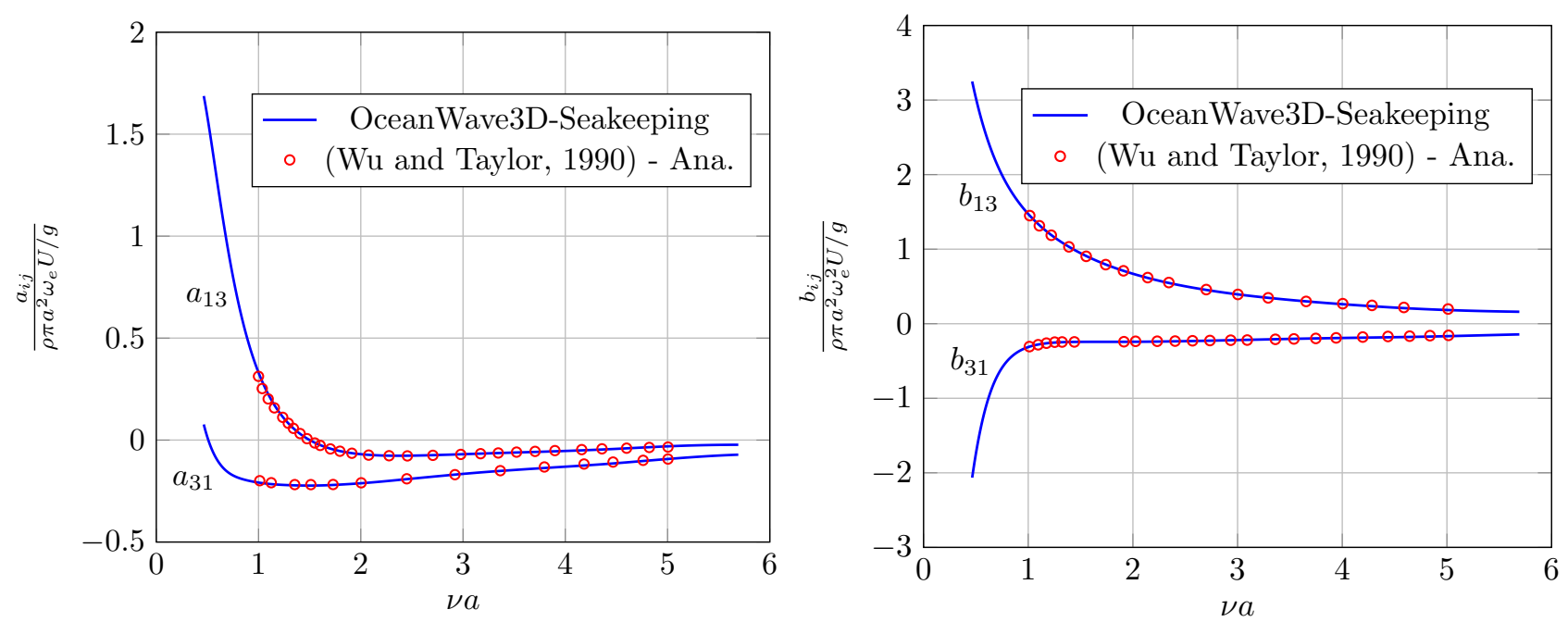

Figure 13: The hydrodynamic coefficients $\left(a_{13}, a_{31}, b_{13}, b_{31}\right)$ for the floating cylinder, $\mathrm{Fr}=0.03$ 

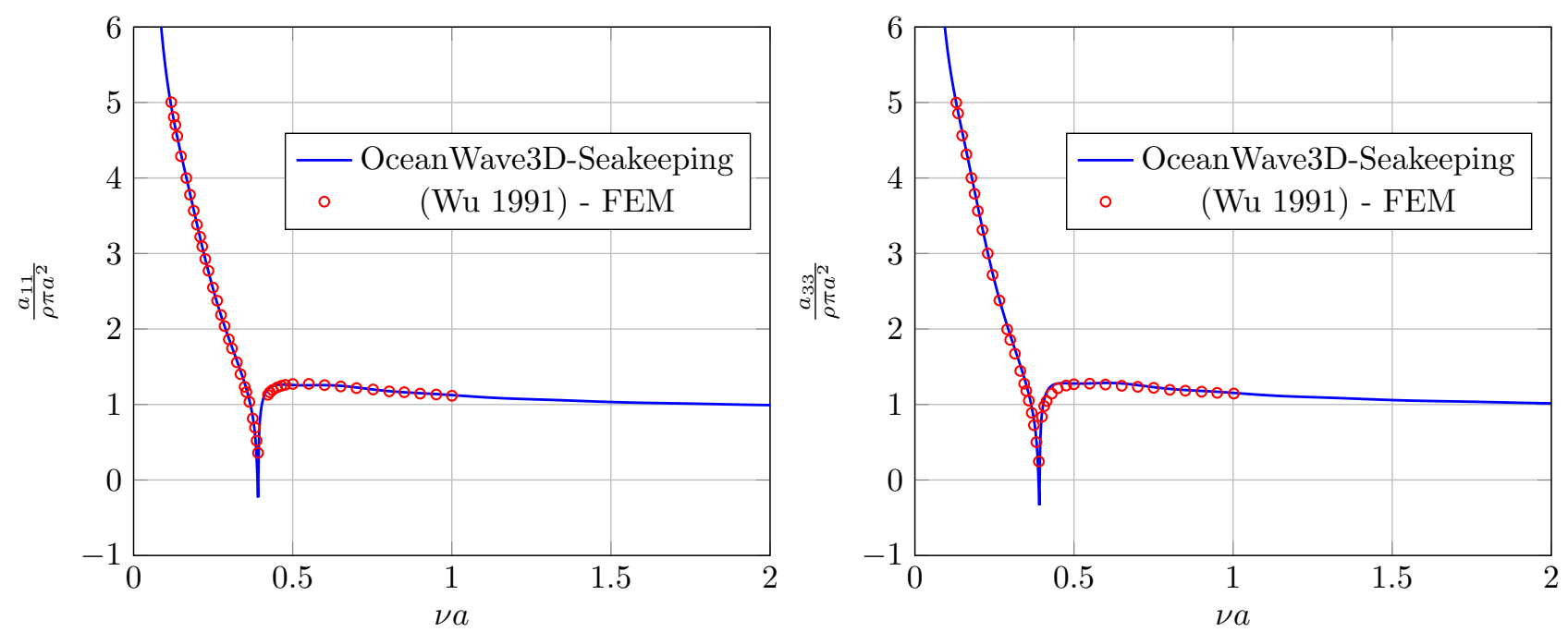

Figure 14: The hydrodynamic coefficients $\left(a_{11}\right.$ and $\left.a_{33}\right)$ for the submerged cylinder, $\operatorname{Fr}=0.4$
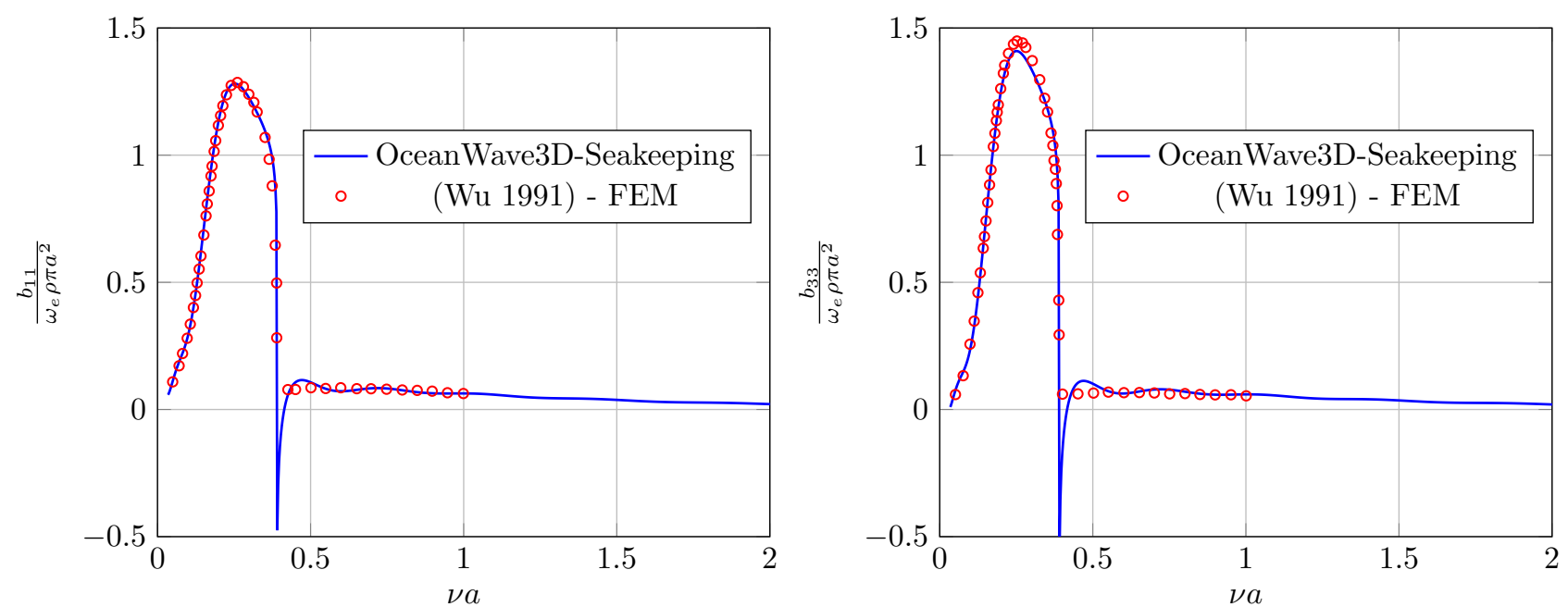

Figure 15: The hydrodynamic coefficients $\left(b_{11}, b_{33}\right)$ for the submerged cylinder, $\mathrm{Fr}=0.4$

\section{The submerged cylinder (High Froude number)}

A relatively high Froude number $(F r=U / \sqrt{g a}=0.4)$ in this case is applied to the submerged circular cylinder. The body oscillates and moves in an infinite depth fluid which is approximated by a discrete domain of depth $d=10 a$. The influence of the critical frequency is relatively large in this case as illustrated in Figure 8. For this set-up, the critical frequency occurs at $\nu_{c} a=0.3906$. The heave and surge added mass and damping coefficients $\left(a_{33}, a_{11}, b_{33}, b_{11}\right)$ are shown in Figures 14 and 15 . The results are compared with the Finite Element Method calculations shown in [31]. 

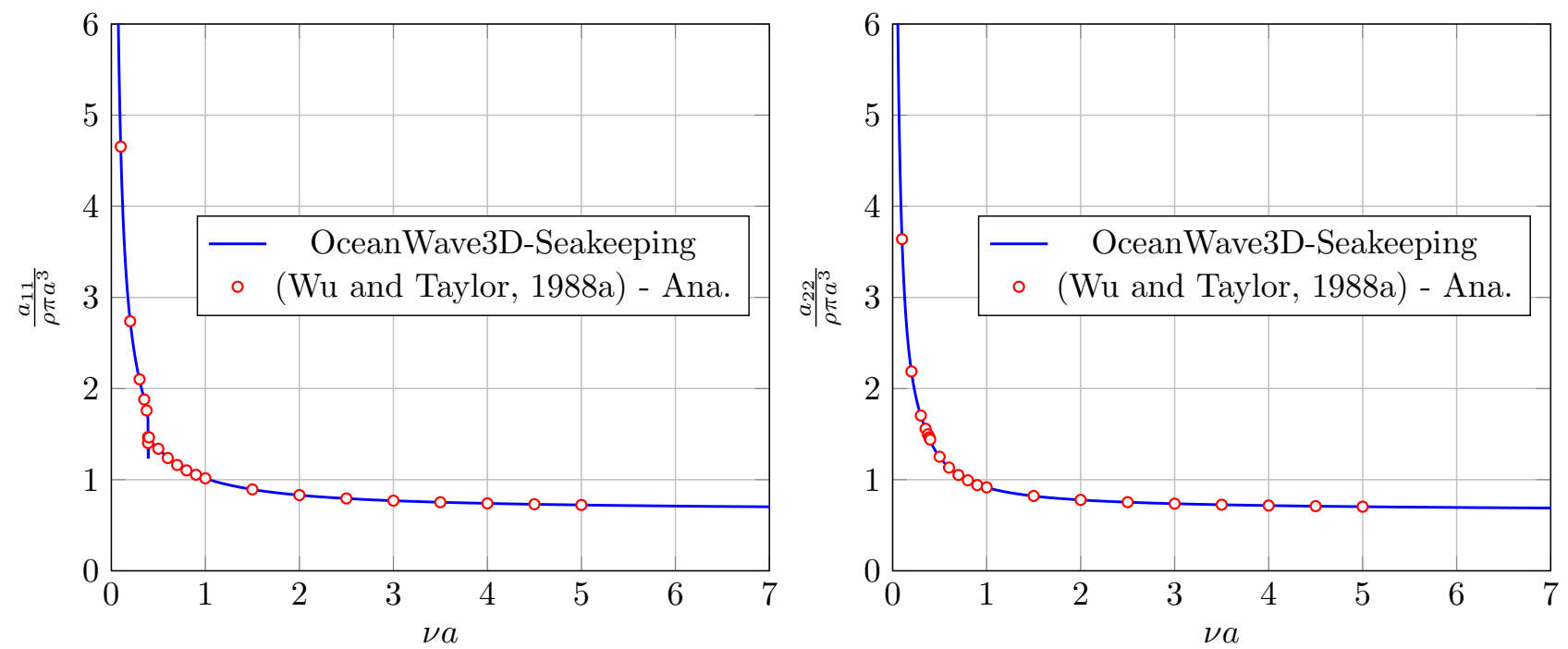

Figure 16: The hydrodynamic coefficients $\left(a_{11}\right.$ and $\left.a_{22}\right)$ for the submerged sphere, $\mathrm{Fr}=0.4$
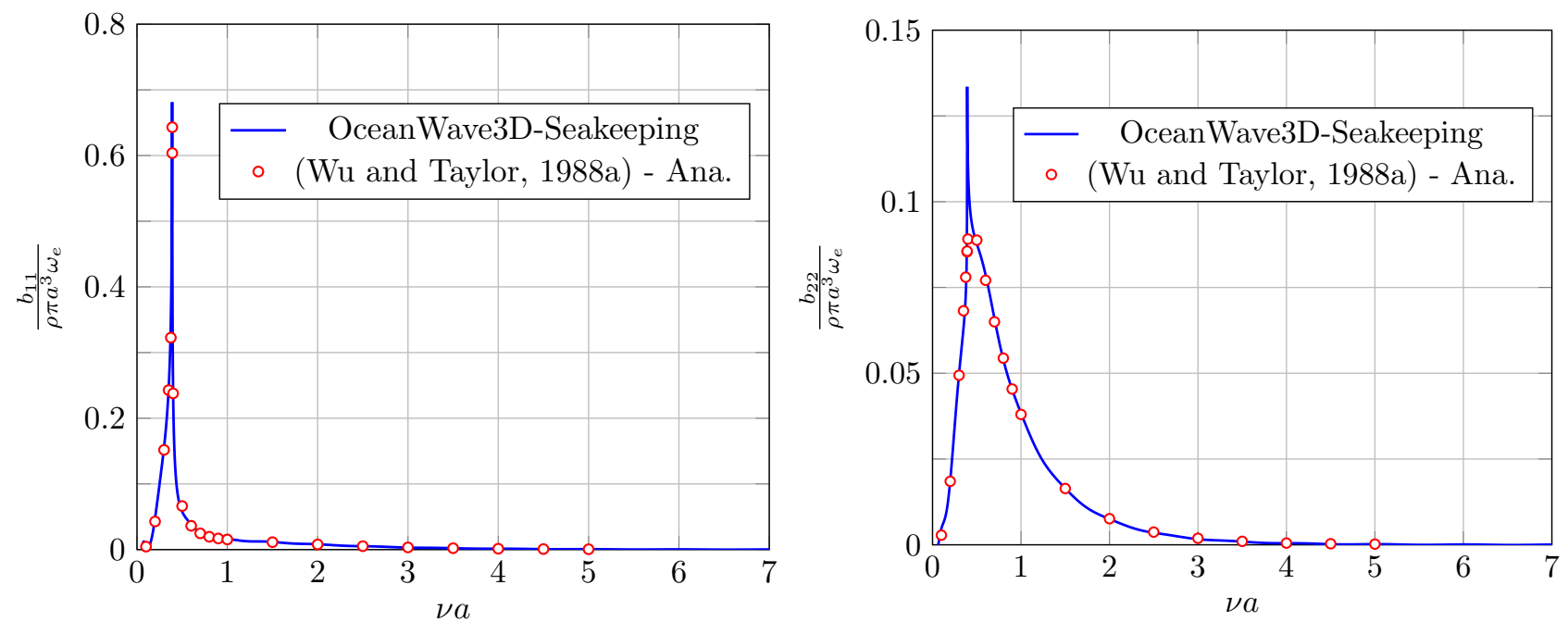

Figure 17: The hydrodynamic coefficients $\left(b_{11}\right.$ and $\left.b_{22}\right)$ for the submerged sphere, $\mathrm{Fr}=0.4$

\section{The submerged sphere}

A three-dimensional test case is prepared for a submerged sphere with radius $a$. The body is moving with a Froude number $F r=U / \sqrt{g a}=0.4$, and the submergence depth is $d=2 a$. The results for the hydrodynamic coefficients are shown in Figures 16-19. The analytical solution for the submerged sphere is due to [32]. In this case the critical frequency occurs at $\nu_{c} a=0.3906$. 

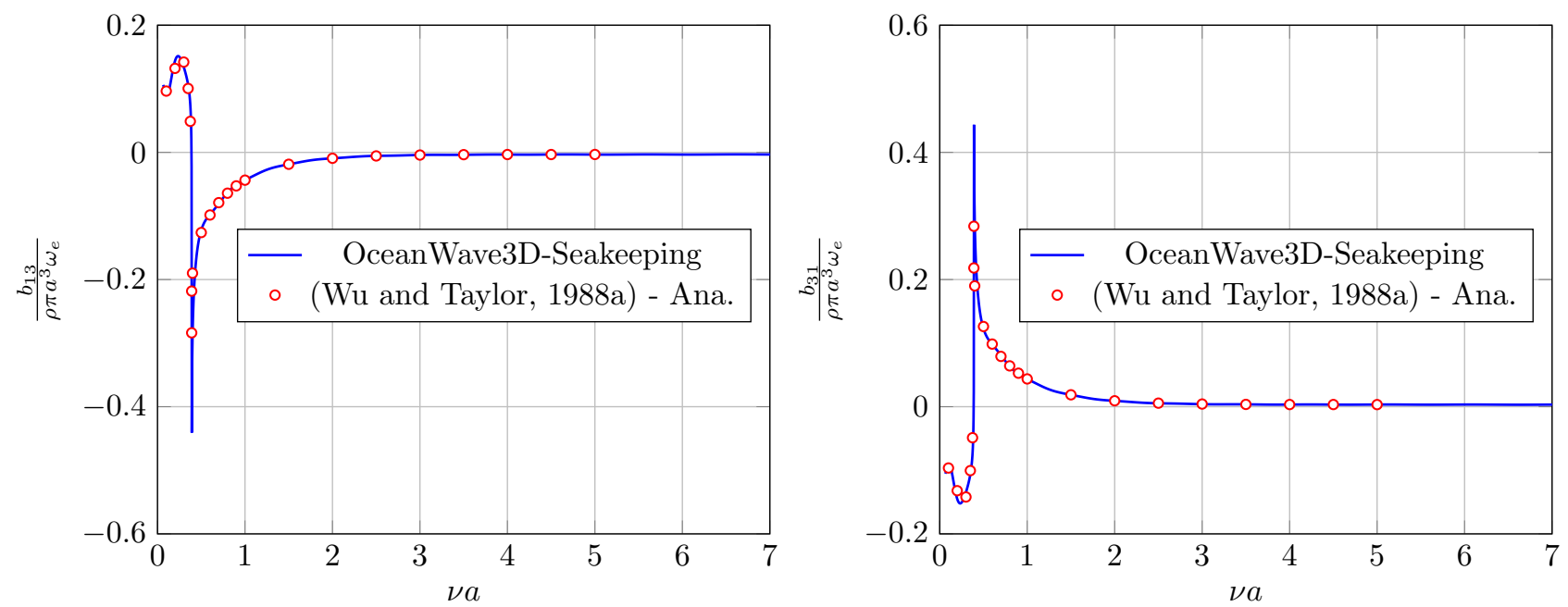

Figure 18: The hydrodynamic coefficients $\left(b_{13}\right.$ and $\left.b_{31}\right)$ for the submerged sphere, $\mathrm{Fr}=0.4$
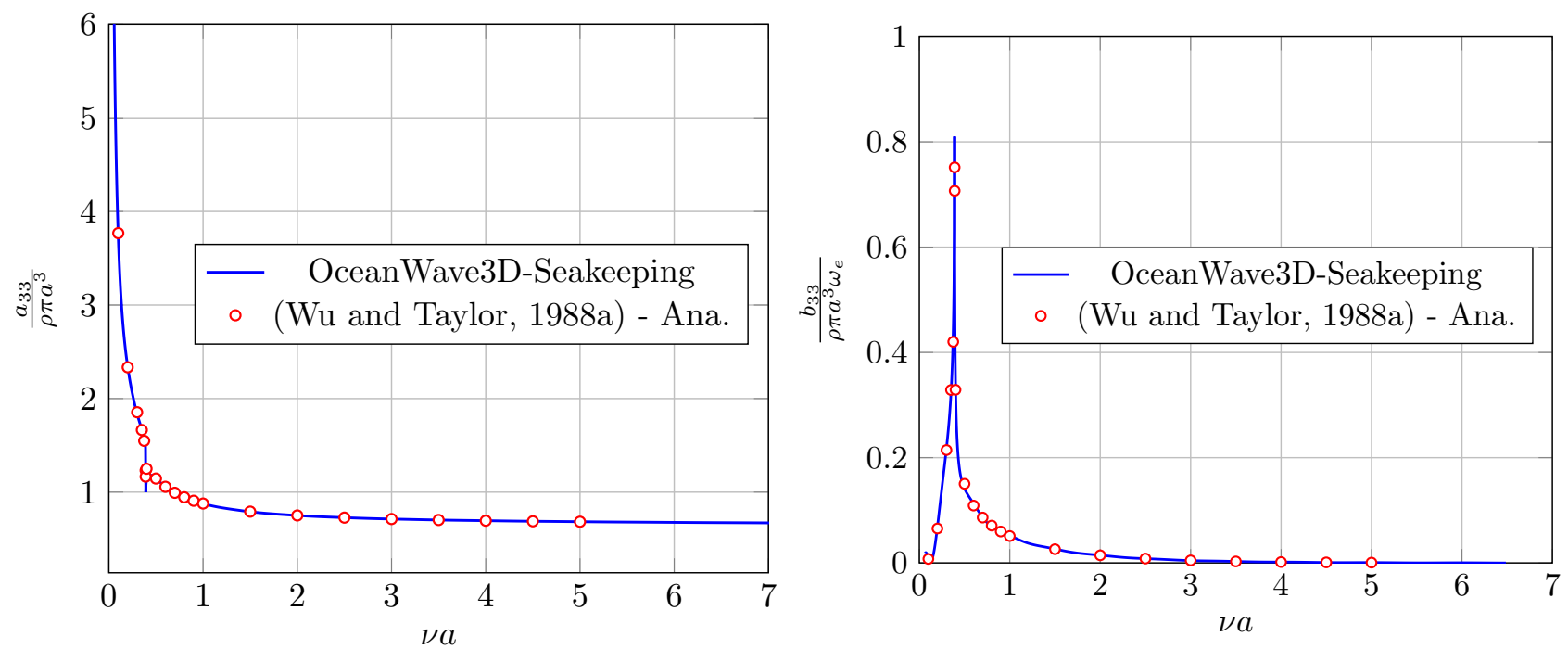

Figure 19: The hydrodynamic coefficients $\left(a_{33}\right.$ and $\left.b_{33}\right)$ for the submerged sphere, $\mathrm{Fr}=0.4$
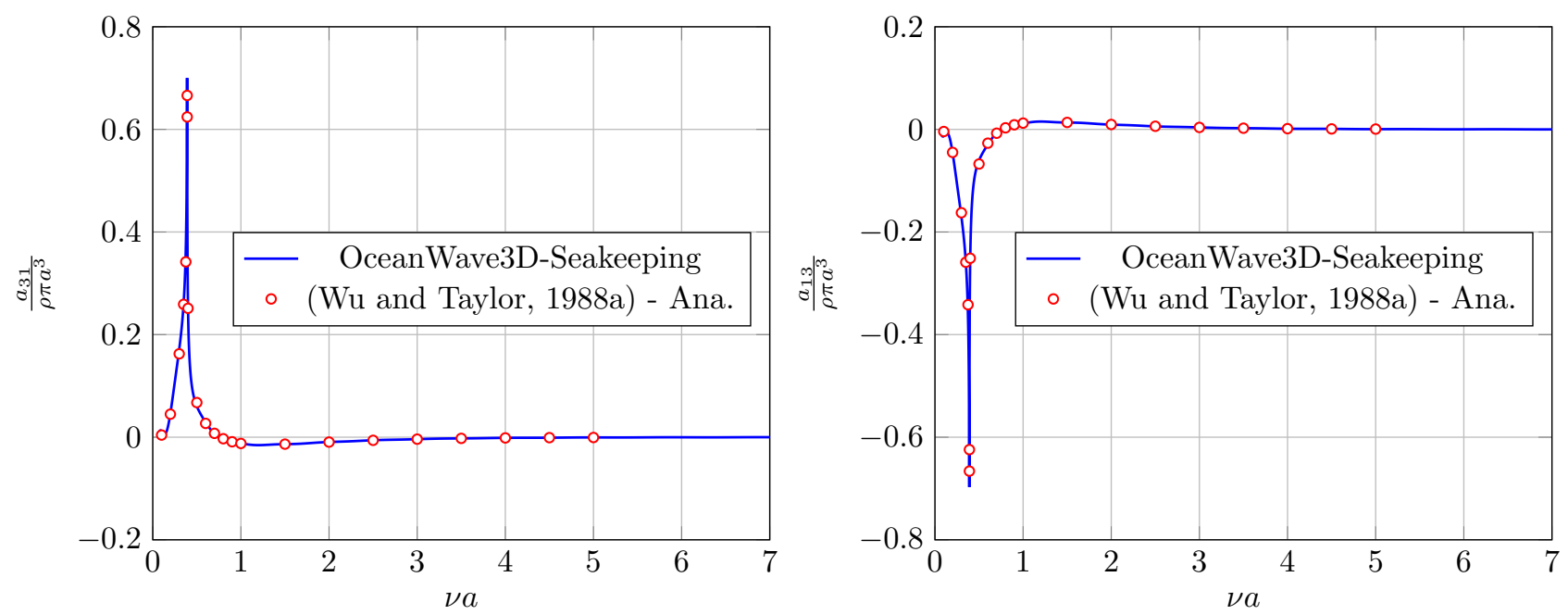

Figure 20: The hydrodynamic coefficients $\left(a_{31}\right.$ and $\left.a_{13}\right)$ for the submerged sphere, $\mathrm{Fr}=0.4$ 


\subsubsection{Discussion of the results}

The numerical calculations for the closed-form geometries (cylinder and sphere) are in excellent agreement with the corresponding analytical and numerical reference solutions. In the case of the hydrodynamic coefficients for the submerged cylinder shown in Figure 15, there are minor differences between the two numerical solutions very close to $\tau=1 / 4$. This is presumably caused by the slightly different methods applied to treat the singularity at the critical frequency. In the deep-water case for analytical solutions, the numerical results at the very low frequencies may be influenced by the finite depth of the numerical basin. In order to overcome this issue, a strong grid stretching in the $z$ direction is adopted together with a very large computational depth. The effect of the depth can hardly be seen for example in in Figure 18 for $\nu a<0.5$. The same stretching strategy is also used at the free surface, so very long waves are not resolved towards the end of the computational domain $S_{\infty}$. This will reduce the adverse effect of the domain size on the results in the very low frequencies.

\subsection{The ship geometry}

As a final more practically relevant test case, an overlapping grid is made for a ship geometry (a bulk carrier). The method of hyperbolic grid generation is employed to generate the component grids [33]. According to this method, first a surface grid is made by a numerical solution to a set of hyperbolic equations governing the grid lines. Next the surface grid is marched in to the volume through the same approach, i.e solving a new set of hyperbolic equations numerically. This will produce the final volume grid around the desired part of the geometry. Generally an arbitrary number of body-fitted component grids can be generated using the hyperbolic method to represent the geometry. For a conventional ship hull, we generally find that three components are sufficient to build a volume grid around the body. Two component grids are defined to represent the ship hull at the fore and aft regions. The third component covers the mid-section of the ship hull with sufficient overlap with the fore and aft end grids. An example of the surface and volume grids for different parts of the bulk carrier is shown in Figures 21 to 23.

The prepared volume grids for the ship hull are then inserted into a background cylindrical grid. The outer limit of this background grid represents the far-field truncation boundary of the computational domain. The final overlapping grid is generated using the overset grid generator Ogen [21]. Part of this overlapping grid can be seen in Figure 24. A good practice is to use very refined grid transformations during the hyperbolic grid generation, which can then be reduced considerably when building the final overset grid. In addition, a high grid resolution is only desirable close to the body surface. Thus a very strong grid stretching towards the far-field boundary is employed. Using these two strategies, the computational time can be significantly reduced.

For the ship geometry, results based on both the Neumann-Kelvin and double-body linearization are presented. The double-body elevation $\zeta_{b}$ is however neglected from the free-surface conditions, based on the commonly applied assumption that this term is actually of order $\epsilon$. The results for the hydrodynamic coefficients due to the forced heave and pitch motions of the bulker are shown in Figures 25-28.

Calculations using the time-domain Green function boundary element solver TiMIT [25] and the strip theory method are also included for reference. Also shown in the figures are the measurements carried out by [34]. The submerged volume of the ship is denoted by $V$, and the body has a Froude number of $F r=U / \sqrt{g L_{p p}}=0.18$, where the length between the perpendiculars is $L_{p p}$. At the critical frequency $\tau=1 / 4$, the value of the non-dimensional wave number $\nu L_{p p}$ is equal to 1.89 . This limit is marked by the gray dashed line in the figures. Based on the finite horizontal domain size, the minimum possible frequency in these computations corresponds to the $\nu_{\min } L_{p p}=0.29$ which is also marked with dotted line. 


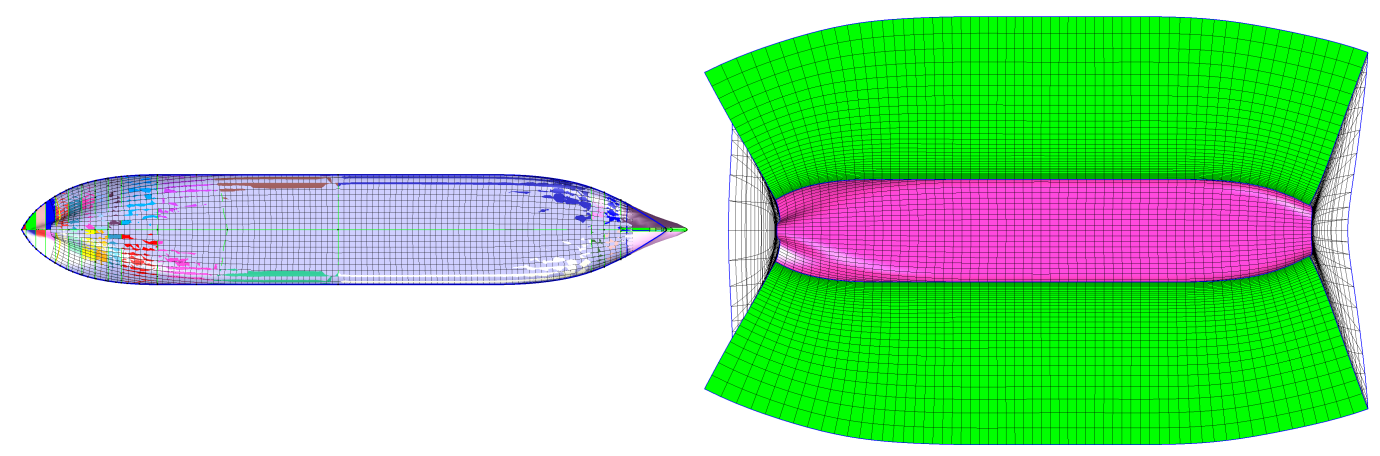

Figure 21: The surface and volume grid for the main body of the ship hull (top view)
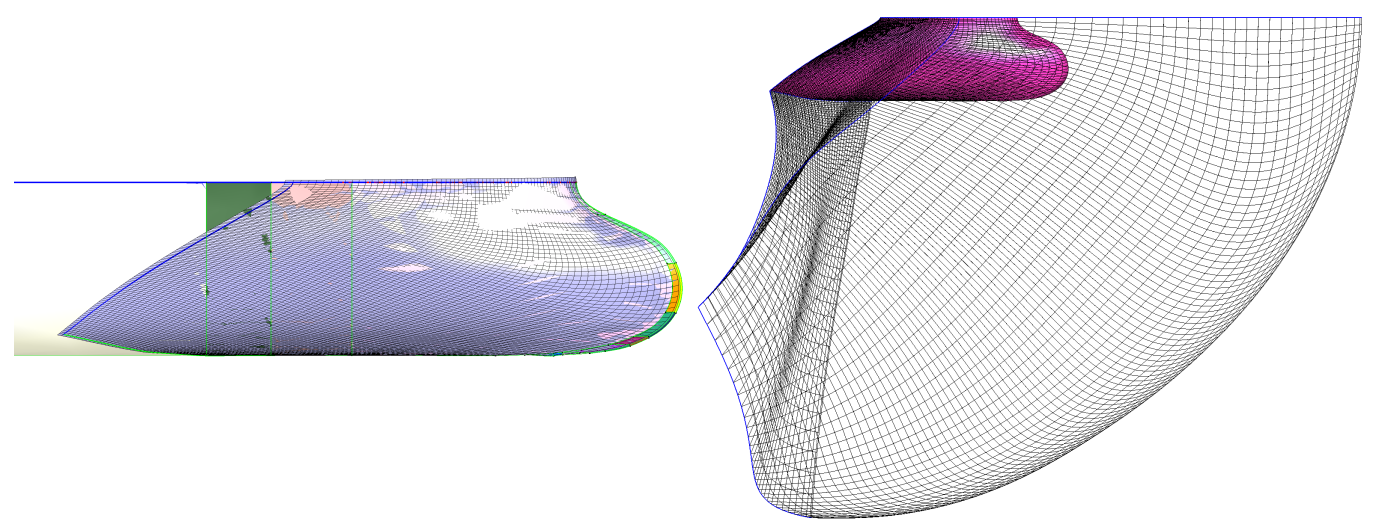

Figure 22: The surface and volume grid for the fore region of the ship hull (side view)
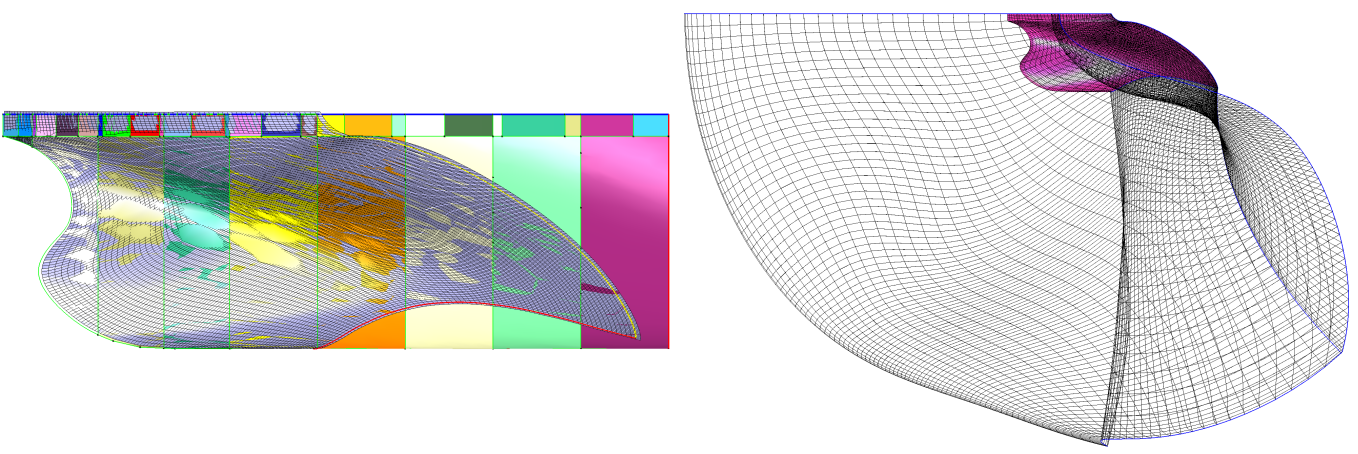

Figure 23: The surface and volume grid for the aft region of the ship hull (side view)

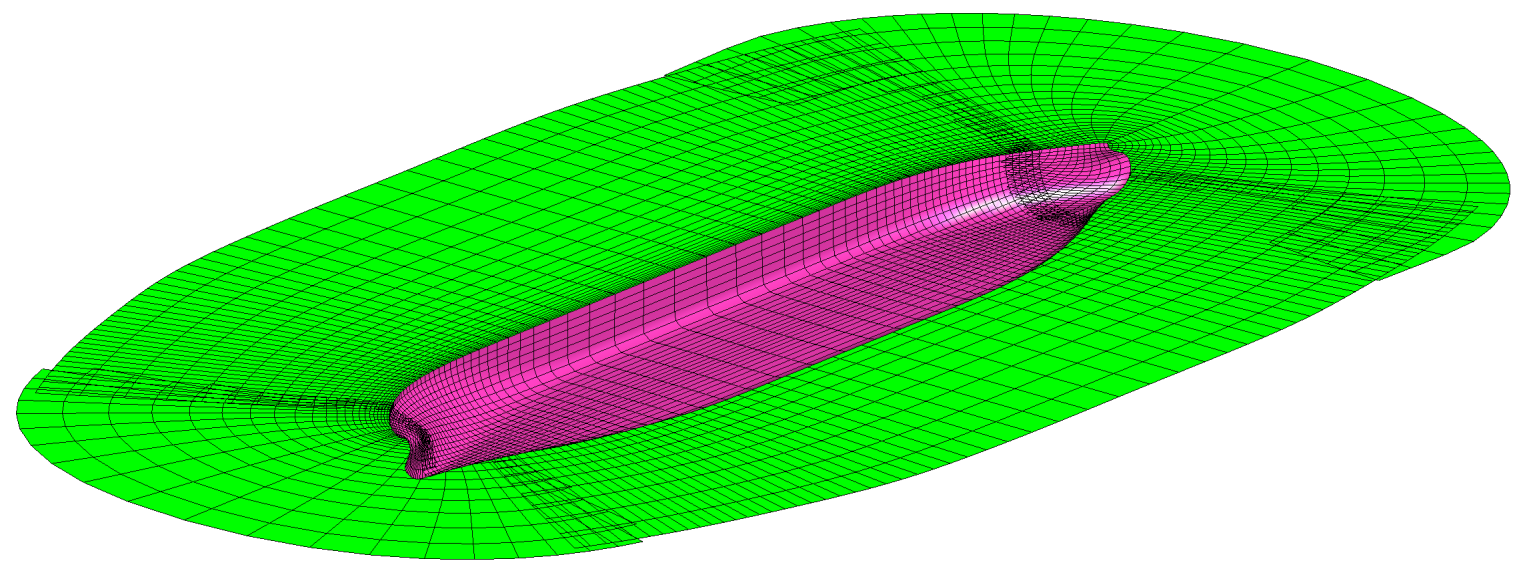

Figure 24: Part of the final overlapping grid for the ship hull (fish view) 

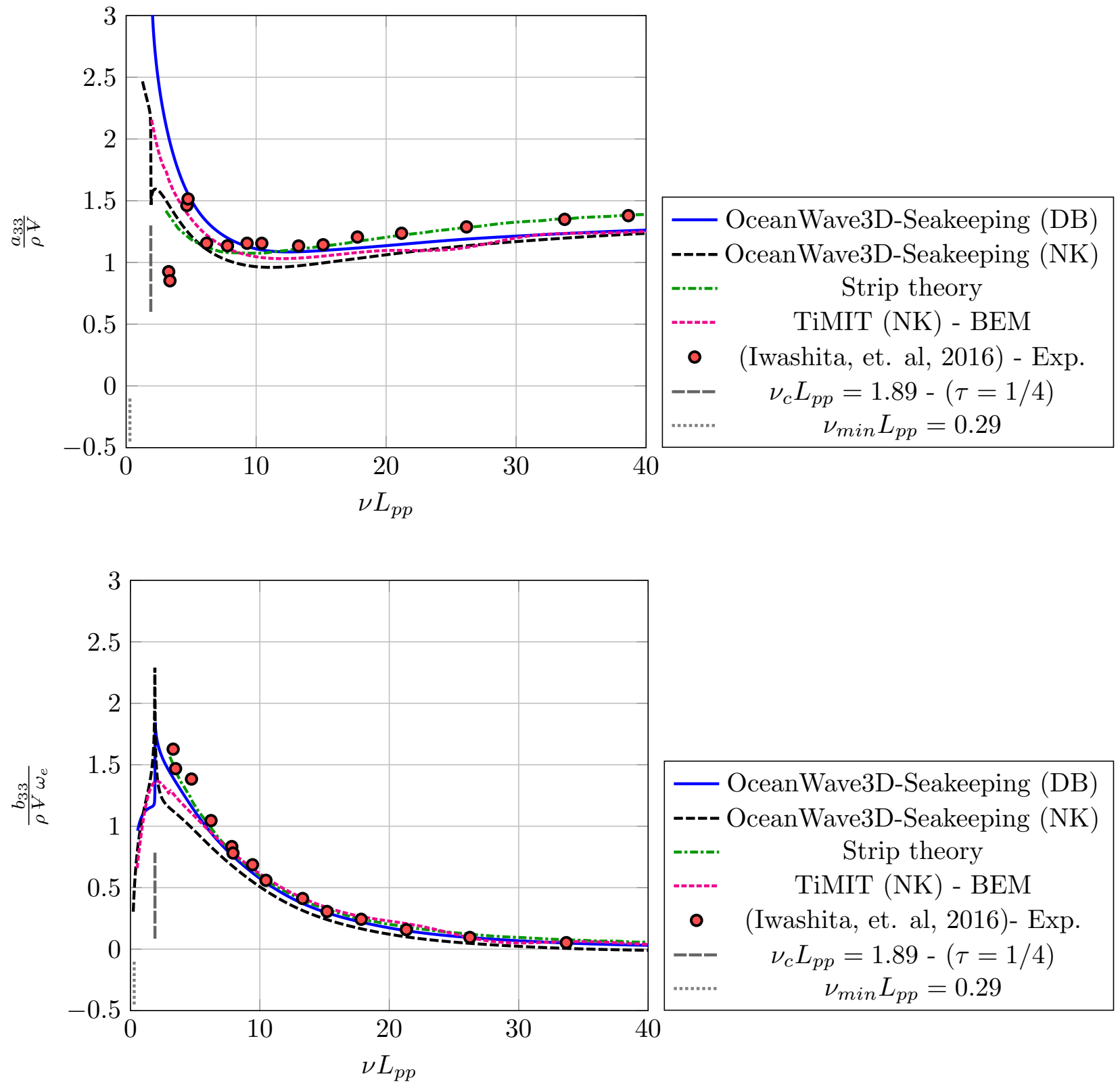

Figure 25: The hydrodynamic coefficients $\left(a_{33}\right.$ and $\left.b_{33}\right)$ for the bulker, Fr $=0.18$ 

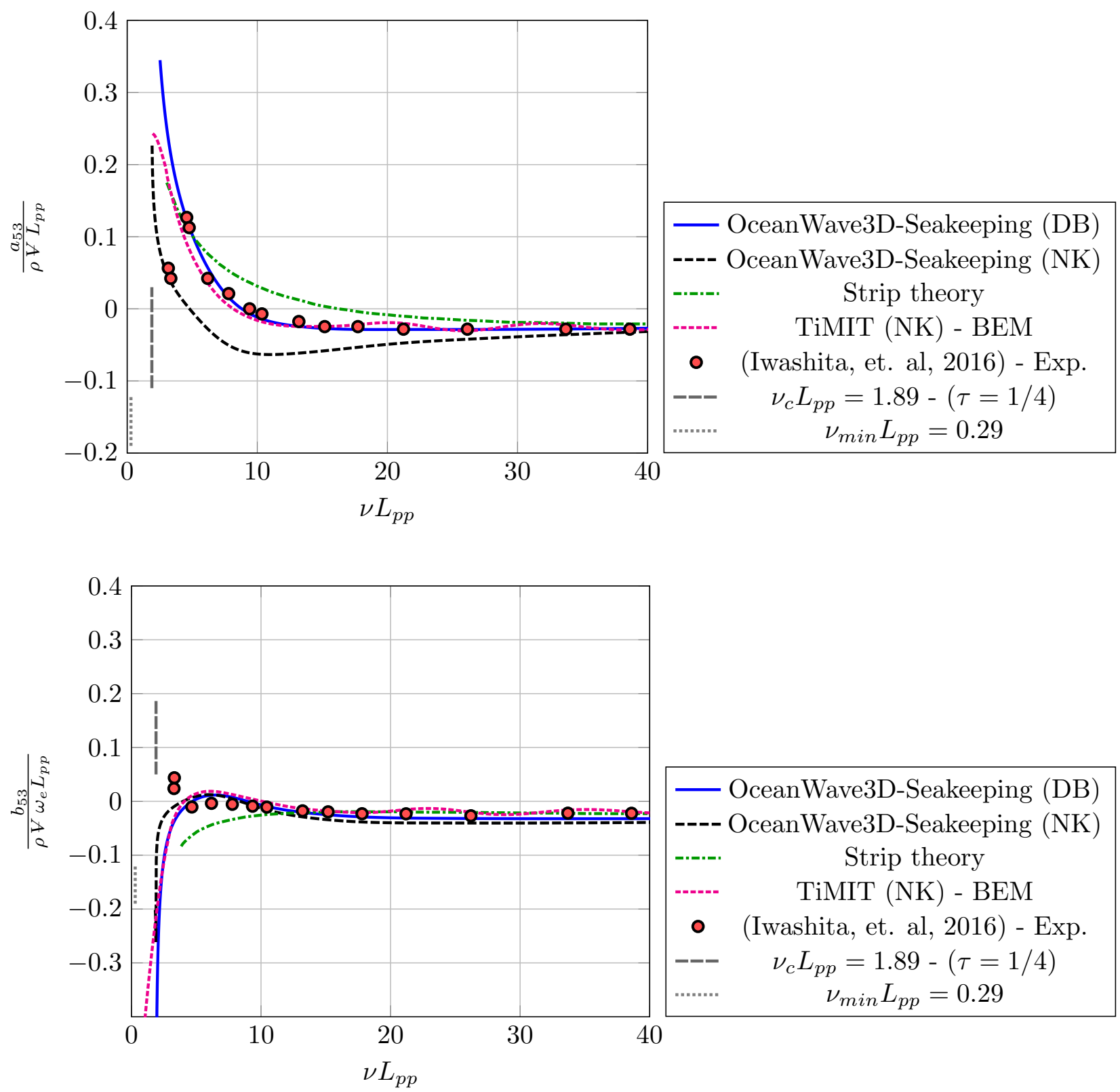

Figure 26: The hydrodynamic coefficients $\left(a_{53}\right.$ and $\left.b_{53}\right)$ for the bulker, Fr $=0.18$ 

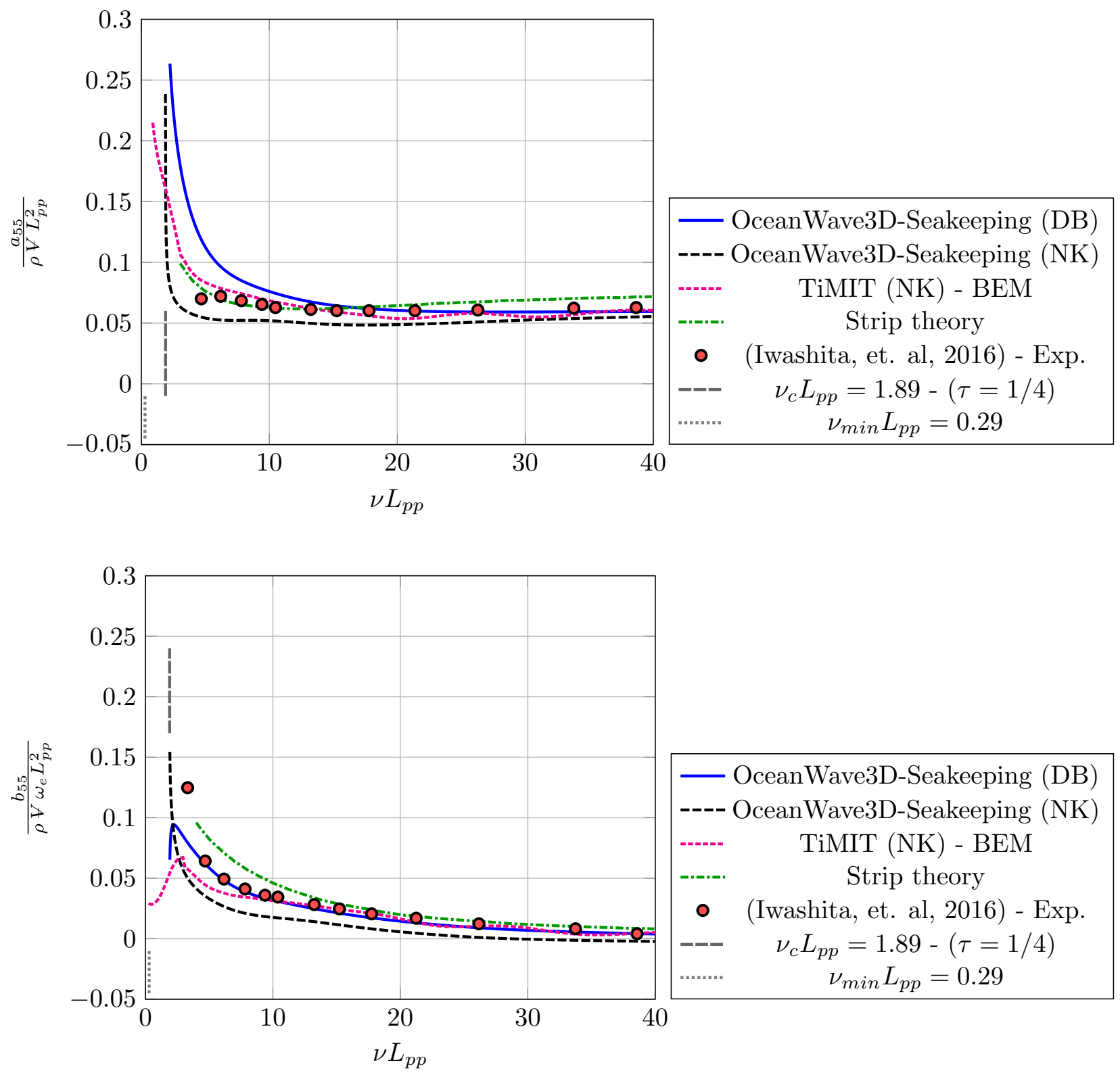

Figure 27: The hydrodynamic coefficients $\left(a_{55}\right.$ and $\left.b_{55}\right)$ for the bulker, Fr $=0.18$ 

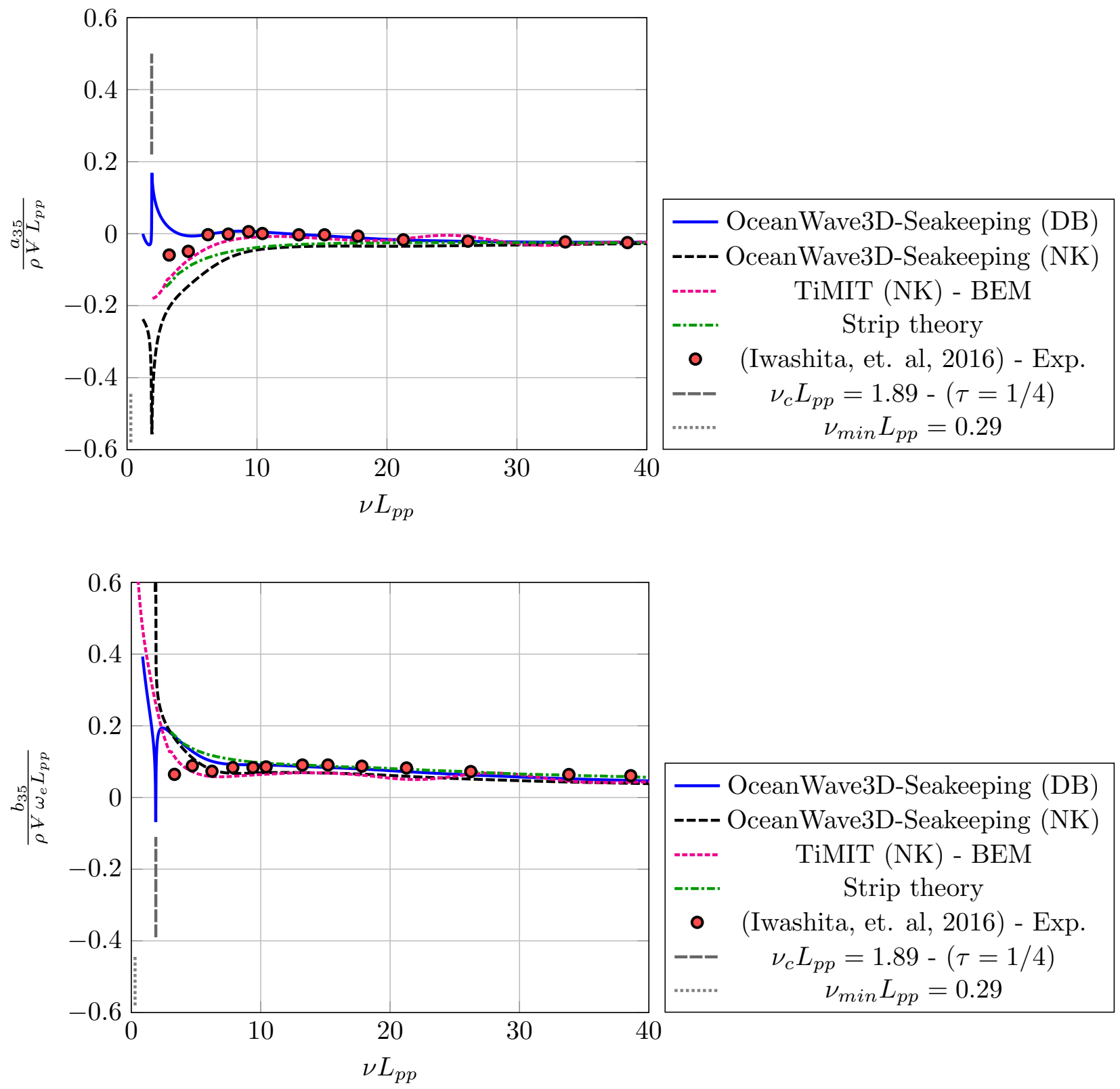

Figure 28: The hydrodynamic coefficients $\left(a_{35}\right.$ and $\left.b_{35}\right)$ for the bulker, Fr $=0.18$ 


\subsubsection{Discussion of the results}

The hydrodynamic coefficients for the bulker based on the double-body linearization compare well with the experimental measurements. With the Neumann-Kelvin linearization, the agreement is generally not as good, which might be expected for a full-form ship. We note however, there are some issues with grid generation for this approach which we are working on improving. In particular, in order to ensure accurate higher derivatives near the body, we are currently forced to slightly modify the hull near the waterline so that it intersects the free surface vertically, as can be seen for example in Figure 23. In addition, the second derivative term at the kinematic free-surface condition is dropped for the bulker simulations, as it could not be calculated with the required accuracy using the current grid generation technique. It is expected that the results for the real ship geometries would be improved by overcoming these issues. More robust and streamlined grid generation strategies are under investigation.

It should also be mentioned that the computational time using the presented finite-difference framework is an order of magnitude longer than the computational time for TIMIT, which is itself an order of magnitude larger than the strip theory calculations. Work is in progress to exploit the parallel processing capabilities of the finite-difference framework to improve the efficiency of the computations. Moreover at the moment a high grid quality using this solver can be achieved only by using a large number of grid points close to the surface of the body. By improving the grid generation for the ship hull, it is expected that a lower number of grid points will be required to get the convergent results. These measures together with the use of an optimized iterative solver and preconditioner would improve the computational efficiency of this solver. It should be also emphasized that we do not expect to be able to compete with well-established time-domain Green function methods for solving the Neumann-Kelvin linearized seakeeping problem. The strength of this finite-difference framework lies in its flexibility to treat more sophisticated linearized formulations, and in the long-term, fully nonlinear problems. In addition, the linear scalability of the computational time by this framework is desirable especially for the prediction of the second-order drift forces (added resistance), which requires much finer resolution than the linear quantities.

\section{Conclusions}

We have presented a high-order finite difference method on overlapping grids for solving the linearized forward-speed radiation problem in the time domain. The stability of the numerical scheme is ensured by employing an upwind biased stencil for the convective derivatives and imposing extrapolation and Neumann conditions at the outflow and the inflow boundaries respectively.

A pseudo-impulsive Gaussian forcing is imposed which is tuned to the resolution of the discrete numerical grid. The influence of the critical frequency is handled by extrapolation of the solution using a least-squares fit to the assumed asymptotic form. The resulting analytical transform is then combined with the numerical transform of the computed signal. The developed solver has been validated using reference solutions from the literature. The expected fourth-order convergence rate with grid resolution has been confirmed, as has the linear scaling of the solution effort with increasing number of unknowns. The solver has also been applied to a bulker hull geometry and a good comparison with experimental measurements was found.

Follow-up papers are in preparation which describe how the method is applied to solve the wave resistance and diffraction problems, and work is in progress to address nonlinear seakeeping.

\section{Acknowledgement}

The authors would like to thank the The Danish Maritime Fund (Den Danske Maritime Fond) for their financial support (No. 2014-088). A special thanks goes also to Prof. M. Kashiwagi from the Department of Naval Architecture \& Ocean Engineering, Osaka University and Prof. H. Iwashita for providing the experimental data and the strip theory results for the bulker. The authors are also 
very grateful to Prof. William D. Henshaw at the Department of Mathematical Sciences, Rensselaer Polytechnic Institute, for his helps regarding the Overture library. 


\section{References}

[1] C.-H. Lee, WAMIT theory manual. Massachusetts Institute of Technology, Department of Ocean Engineering, 1995.

[2] W. Cummins, "The impulse response function and ship motions," tech. rep., DTIC Document, 1962.

[3] T. Ogilvie, "Recent progress towards the understanding and prediction of ship motions," in Proceedings of the 5th Symposium on Naval Hydrodynamics,1964., 1964.

[4] R. F. Beck and A. R. Magee, "Time-domain analysis for predicting ship motions," in Proceedings of IUTAM Symposium, Dynamics of Marine Vehicles and Structures in Waves, (London), 1990.

[5] H. B. Bingham, Simulating ship motions in the time domain. PhD thesis, Massachusetts Institute of Technology, Department of Ocean Engineering, 1994.

[6] D. Nakos, Ship wave patterns and motions by a three dimensional Rankine panel method. PhD thesis, Massachusetts Institute of Technology, 1990.

[7] D. C. Kring, Time domain ship motions by a three-dimensional Rankine panel method. PhD thesis, Massachusetts Institute of Technology, 1994.

[8] H. B. Bingham and H. Zhang, "On the accuracy of finite-difference solutions for nonlinear water waves," Journal of Engineering Mathematics, vol. 58, no. 1-4, pp. 211-228, 2007.

[9] A. P. Engsig-Karup, O. Lindberg, S. L. Glimberg, B. Dammann, H. B. Bingham, and P. A. Madsen, "A high-order weno finite difference water wave model for interactive ship-wave simulation," in International Conference on Spectral and High Order Methods (ICOSAHOM 2012), 2012.

[10] A. P. Engsig-Karup, H. B. Bingham, and O. Lindberg, "An efficient flexible-order model for 3d nonlinear water waves," Journal of computational physics, vol. 228, no. 6, pp. 2100-2118, 2009.

[11] J. White, J. R. Phillips, and F. T. Korsmeyer, "Comparing precorrected-FFT and fast multipole algorithms for solving three-dimensional potential integral equations," in The Colorado conference in iterative methods, (Breckenridge, Colorado), 1994.

[12] S.-c. Jiang, B. Teng, Y. Gou, and D.-z. Ning, "A precorrected-fet higher-order boundary element method for wave-body problems," Engineering Analysis With Boundary Elements, vol. 36, no. 3, pp. 404-415, 2012.

[13] M. Amini Afshar, Towards Predicting the Added Resistance of Slow Ships in Waves. PhD thesis, DTU Mechanical Engineering, 2015.

[14] M. Amini Afshar, H. B. Bingham, W. Henshaw, and R. Read, "Convergence of near-field added resistance calculations using a high-order finite-difference method," in Proceedings of 13th PRADS, 2016.

[15] S. J. Liapis, Time-domain analysis of ship motion. PhD thesis, The University of Michigan, 1986.

[16] B. King, "Time-domain analysis of wave exciting forces on ships and bodies," tech. rep., University of Michigan, 1987.

[17] H. B. Bingham, F. T. Korsmeyer, J. N. Newman, and G. E. Osborne, "The simulation of ship motions," in $6^{\text {th }}$ Intern. Conference on Numerical Ship Hydrodynamics, (Iowa City, Iowa), National Academy of Sciences, USA, 1993. 
[18] J. Newman, "The theory of ship motions," Advances in Applied Mechanics, vol. 18, pp. 221-283, 1979.

[19] R. J. LeVeque, Finite difference methods for ordinary and partial differential equations: steadystate and time-dependent problems, vol. 98. Siam, 2007.

[20] D. L. Brown, W. D. Henshaw, and D. J. Quinlan, "Overture: An object-oriented framework for solving partial differential equations on overlapping grids," Object Oriented Methods for Interoperable Scientific and Engineering Computing, SIAM, pp. 245-255, 1999.

[21] W. D. Henshaw, "Ogen: An overlapping grid generator for overture," LANL unclassified report, pp. 96-3466, 1998.

[22] W. Henshaw, "Finite difference operators and boundary conditions for overture, user guide," tech. rep., version 1.00. Technical Report LA-UR-96-3467, Los Alamos National Laboratory, 1998.

[23] W. D. Henshaw, Part I. The numerical solution of hyperbolic systems of conservation laws. Part II. Composite overlapping grid techniques. PhD thesis, California Institute of Technology, 1985.

[24] J. N. Newman, Marine Hydrodynam. The MIT press, 1977.

[25] F. Korsmeyer, H. Bingham, and J. Newman, TiMIT - A Panel Method for Transient Wave-Body Interactions. Research Laboratory of Electronics, MIT, 1999.

[26] J. Grue and E. Palm, "Wave radiation and wave diffraction from a submerged body in a uniform current," Journal of Fluid Mechanics, vol. 151, pp. 257-278, 1985.

[27] O. Faltinsen, Sea loads on ships and offshore structures, vol. 1. Cambridge University Press, 1993.

[28] J. V. Wehausen and E. V. Laitone, "Surface waves," in Fluid Dynamics/Strömungsmechanik, pp. 446-778, Springer, 1960.

[29] G. Wu and R. E. Taylor, "Reciprocity relations for the hydrodynamic coefficients of bodies with forward speed," International shipbuilding progress, vol. 35, no. 402, 1988.

[30] G. Wu and R. E. Taylor, "The hydrodynamic force on an oscillating ship with low forward speed," Journal of Fluid Mechanics, vol. 211, pp. 333-353, 1990.

[31] G. Wu, "Hydrodynamic forces on a submerged cylinder advancing in water waves of finite depth," Journal of Fluid Mechanics, vol. 224, pp. 645-659, 1991.

[32] G. Wu and R. E. Taylor, "Radiation and diffraction of water waves by a submerged sphere at forward speed," Proceedings of the Royal Society of London. A. Mathematical and Physical Sciences, vol. 417, no. 1853, pp. 433-461, 1988.

[33] W. M. Chan, "Hyperbolic methods for surface and field grid generation," in Handbook of Grid Generation (S. Thompson and Weatherill, eds.), ch. 5, pp. 5-1-5-26, CRC Press, 1998.

[34] H. Iwashita, M. Kashiwagi, Y. Ito, and Y. Seki, "Calculations of ship seakeeping in low-speed/lowfrequency range by frequency-domain rankine panel methods," Journal of the Japan Society of Naval Architects and Ocean Engineers, vol. 24, pp. 129-146, 2016, (In Japanese).

[35] R. G. Dean and R. A. Dalrymple, Water wave mechanics for engineers and scientists. World Scientific., 1991.

[36] T. F. Ogilvie, "Second-order hydrodynamic effects on ocean platforms," in International Workshop on Ship and Platform Motions, University of California, Berkeley, 1983. 
[37] C. C. Mei, M. Stiassnie, and D. K.-P. Yue, Theory and applications of ocean surface waves: linear aspects, vol. 23. World Scientific, 2005.

[38] T. Ogilvie and E. Tuck, "A rational strip theory for ship motions, part 1," Dept. Naval Archit., University of Michigan, Rep, 1969.

[39] J. Newman, "Wave effects on deformable bodies," Applied Ocean Research, vol. 16, no. 1, pp. 47$59,1994$. 


\section{A Nonlinear formulation}

In this Appendix, we briefly review the nonlinear formulation of the seakeeping problem. An earthfixed coordinate system $\left(O_{0}-x_{0}, y_{0}, z_{0}\right)$ is adopted. The continuity equation is governing in the whole domain at each time step. This is prescribed by the Laplacian of the velocity potential as:

$$
\nabla^{2} \Phi=\frac{\partial^{2} \Phi}{\partial x_{0}^{2}}+\frac{\partial^{2} \Phi}{\partial y_{0}^{2}}+\frac{\partial^{2} \Phi}{\partial z_{0}^{2}}=0
$$

As can be seen from Figure 1, there are 4 boundaries, i.e $S_{f}$ at the free surface, $S_{b}$ at the instantaneous surface of the body, $S_{d}$ at the sea bed and $S_{\infty}$ at the far-field truncation boundary of the domain. Appropriate conditions should be defined at these boundaries. Two equations are required to prescribe the kinematic and dynamic conditions at the free surface. The dynamic condition equates the fluid pressure at the free surface $z_{0}=\zeta\left(x_{0}, y_{0}\right)$ to the atmospheric pressure. This can be described by the unsteady form of the Bernoulli equation as follows:

$$
-\frac{p-p_{a}}{\rho}=\frac{\partial \Phi}{\partial t}+\frac{1}{2} \nabla \Phi \cdot \nabla \Phi+g z_{0}=0, \text { at } z_{0}=\zeta\left(x_{0}, y_{0}\right),
$$

where the density of the fluid is $\rho$, and the acceleration due to the gravity is $g$. The atmospheric pressure is $p_{a}$ and the fluid pressure is denoted by $p$.

To derive the kinematic condition of the free surface and also the body boundary condition, a general approach is adopted in this paper [35]. An arbitrary surface in the domain which can be described by an expression like:

$$
S\left(x_{0}, y_{0}, z_{0}, t\right)=0
$$

is considered. Then the total time derivative of the surface would be zero, as the total change of the surface with respect to an observer moving with the surface is zero. This can be expressed by:

$$
\frac{d}{d t} S=\left(\frac{\partial}{\partial t}+\boldsymbol{u} \cdot \nabla\right) S=\frac{\partial S}{\partial t}+\frac{\partial S}{\partial x_{0}} \frac{\partial \Phi}{\partial x_{0}}+\frac{\partial S}{\partial y_{0}} \frac{\partial \Phi}{\partial y_{0}}+\frac{\partial S}{\partial z_{0}} \frac{\partial \Phi}{\partial z_{0}}=0, \quad \text { at } S\left(x_{0}, y_{0}, z_{0}, t\right)=0
$$

in which:

$$
\boldsymbol{u}=\left(\frac{\partial \Phi}{\partial x_{0}}, \frac{\partial \Phi}{\partial y_{0}}, \frac{\partial \Phi}{\partial z_{0}}\right)=\nabla \Phi
$$

As the gradient of the function $S$ is in fact a vector normal to the surface, a unit normal vector for the surface can also be obtained by:

$$
\boldsymbol{n}_{0}=\frac{\nabla S}{|\nabla S|}
$$

So a kinematic condition for the arbitrary surface can be written:

$$
\boldsymbol{u} \cdot \boldsymbol{n}_{0}=-\frac{\partial S}{\partial t} /|\nabla S|
$$

For the free-surface elevation $S=S_{f}=z_{0}-\zeta\left(x_{0}, y_{0}, t\right)=0$, and the relevant kinematic condition is obtained as:

$$
\frac{\partial \Phi}{\partial z_{0}}=\frac{\partial \zeta}{\partial t}+\frac{\partial \Phi}{\partial x_{0}} \frac{\partial \zeta}{\partial x_{0}}+\frac{\partial \Phi}{\partial y_{0}} \frac{\partial \zeta}{\partial y_{0}} \quad \text { at } z_{0}=\zeta\left(x_{0}, y_{0}, t\right)
$$

The same argument is also used for the body boundary condition, where $S=S_{b}\left(x_{0}, y_{0}, z_{0}, t\right)=0$. The corresponding kinematic condition can be obtained as:

$$
\frac{\partial S_{b}}{\partial t}=-\frac{\partial S_{b}}{\partial x_{0}} \frac{\partial \Phi}{\partial x_{0}}-\frac{\partial S_{b}}{\partial y_{0}} \frac{\partial \Phi}{\partial y_{0}}-\frac{\partial S_{b}}{\partial z_{0}} \frac{\partial \Phi}{\partial z_{0}}=\boldsymbol{u} \cdot \boldsymbol{n}_{0} \quad \text { at } S_{b}\left(x_{0}, y_{0}, z_{0}, t\right)=0 .
$$

For the bed and the far-field truncation boundary the relevant boundary condition would be:

$$
\boldsymbol{u} \cdot \boldsymbol{n}_{0}=0, \quad \text { at } S_{d} \text { and } S_{\infty}
$$

as these surfaces are not changing in time. 


\section{B Linearized body boundary condition and m-terms}

\section{B.1 First-order relation between the body-fixed and inertial frames of reference}

In order to get a linearized body boundary condition, first we consider the general relation which can be established between the body-fixed coordinate system $\left(O^{\prime}-x^{\prime}, y^{\prime}, z^{\prime}\right)$ and the inertial translating coordinate system $(O-x, y, z)$ as follows:

$$
\boldsymbol{r}^{\prime}=\mathbf{L}(\boldsymbol{r}-\boldsymbol{\xi}) \quad \text { or } \quad \boldsymbol{r}=\boldsymbol{\xi}+\mathbf{L}^{T} \boldsymbol{r}^{\prime} .
$$

Here $\boldsymbol{\xi}=\left(\xi_{1}, \xi_{2}, \xi_{3}\right)$ corresponding to the surge, sway and heave motions, is the vector connecting the origin of the coordinate systems. $\boldsymbol{r}$ and $\boldsymbol{r}^{\prime}$ are the position vector for an arbitrary point with respect to the inertial and the body-fixed coordinate system. The transformation matrix $\mathbf{L}$ which is unitary (i.e $\mathbf{L}^{T}=\mathbf{L}^{-1}$ ) is defined by:

$$
\mathbf{L}=\left(\begin{array}{ccc}
c_{5} c_{6} & c_{4} s_{6}+s_{4} s_{5} c_{6} & s_{4} s_{6}-c_{4} s_{5} c_{6} \\
-c_{5} s_{6} & c_{4} c_{6}-s_{4} s_{5} s_{6} & s_{4} c_{6}+c_{4} s_{5} s_{6} \\
s_{5} & -s_{4} c_{5} & c_{4} c_{5}
\end{array}\right)
$$

in which $c_{i}=\cos \left(\xi_{i}\right)$ and $s_{i}=\sin \left(\xi_{i}\right)$. Note that the order of the transformation is given by roll $\left(\xi_{4}=\Omega_{1}\right)$, pitch $\left(\xi_{5}=\Omega_{2}\right)$ and yaw $\left(\xi_{6}=\Omega_{3}\right)$ respectively. A first-order approximation to $\mathbf{L}$ denoted by $\hat{\mathbf{L}}$ can be obtained as:

$$
\hat{\mathbf{L}}=\left[\begin{array}{ccc}
1 & \Omega_{3} & -\Omega_{2} \\
-\Omega_{3} & 1 & \Omega_{1} \\
\Omega_{2} & -\Omega_{1} & 1
\end{array}\right]
$$

which is a combination of the following three transformation matrix for each corresponding rotation:

$$
\hat{\boldsymbol{L}}_{\Omega_{1}}=\left[\begin{array}{ccc}
1 & 0 & 0 \\
0 & 1 & \Omega_{1} \\
0 & -\Omega_{1} & 1
\end{array}\right], \quad \hat{\boldsymbol{L}}_{\Omega_{2}}=\left[\begin{array}{ccc}
1 & 0 & -\Omega_{2} \\
0 & 1 & 0 \\
\Omega_{2} & 0 & 1
\end{array}\right], \quad \hat{\boldsymbol{L}}_{\Omega_{3}}=\left[\begin{array}{ccc}
1 & \Omega_{3} & 0 \\
-\Omega_{3} & 1 & 0 \\
0 & 0 & 1
\end{array}\right] .
$$

In fact up to the first order, the final transformation matrix $\hat{\mathbf{L}}$ can be obtained by an arbitrary order product of $\hat{\mathbf{L}}_{\Omega_{1}}, \hat{\mathbf{L}}_{\Omega_{2}}$ and $\hat{\mathbf{L}}_{\Omega_{3}}$ matrices. The linearized transformation matrix is inserted in to (B.1) to obtain up to the first order:

$$
\left[\begin{array}{l}
x^{\prime} \\
y^{\prime} \\
z^{\prime}
\end{array}\right]=\left(x-\xi_{1}\right)\left[\begin{array}{c}
1 \\
-\Omega_{3} \\
\Omega_{2}
\end{array}\right]+\left(y-\xi_{2}\right)\left[\begin{array}{c}
\Omega_{3} \\
1 \\
-\Omega_{1}
\end{array}\right]+\left(z-\xi_{3}\right)\left[\begin{array}{c}
-\Omega_{2} \\
\Omega_{1} \\
1
\end{array}\right]=\left[\begin{array}{l}
x-\xi_{1}+y \Omega_{3}-z \Omega_{2} \\
y-\xi_{2}-x \Omega_{3}+z \Omega_{1} \\
z-\xi_{3}+x \Omega_{2}-y \Omega_{1}
\end{array}\right] .
$$

Or equivalently:

$$
\begin{aligned}
& \alpha_{1}=x-x^{\prime}=\xi_{1}+\Omega_{2} z-\Omega_{3} y, \\
& \alpha_{2}=y-y^{\prime}=\xi_{2}+\Omega_{3} x-\Omega_{1} z, \\
& \alpha_{3}=z-z^{\prime}=\xi_{3}+\Omega_{1} y-\Omega_{2} x .
\end{aligned}
$$

Note that as this linear transformation is commutable, it is allowed to regard $\boldsymbol{\Omega}$ as a vector and write the above relation also as:

$$
\alpha=r-r^{\prime}=\xi+\Omega \times r .
$$

Second- and higher-order relation can also be established between these coordinate systems as has been described by [36]. 


\section{B.2 Linearization of the body boundary condition}

The instantaneous surface of the body is denoted by $S_{b}(x, y, z, t)$. To first order, the instantaneous body surface can be expressed by:

$$
S_{b}(x, y, z, t)=S_{0}(x, y, z)+S_{u}(x, y, z, t),
$$

where $S_{0}$ describes the initial undisturbed surface of the body, and $S_{u}$ defines the time-dependent first order surface of the body. The velocity potential from (2.2) and the body surface from (B.6) can be inserted in to the nonlinear body boundary condition (A.9) to get:

$$
\frac{\partial S_{u}}{\partial t}+\nabla S_{0} \cdot \nabla \phi_{b}+\nabla S_{u} \cdot \nabla \phi_{b}+\nabla S_{0} \cdot \nabla \phi=0 \quad \text { at } S_{b}(x, y, z, t)=0 .
$$

The above boundary condition has been expressed on the exact position of the body. Using Taylor expansion, this condition can be transferred to the mean body surface $S_{0}$ as follows:

$$
\begin{array}{r}
\left.\left(\frac{\partial S_{u}}{\partial t}+\nabla S_{0} \cdot \nabla \phi_{b}+\nabla S_{u} \cdot \nabla \phi_{b}+\nabla S_{0} \cdot \nabla \phi\right)\right|_{S_{b}}=\left.\left(\frac{\partial S_{u}}{\partial t}+\nabla S_{u} \cdot \nabla \phi_{b}+\nabla S_{0} \cdot \nabla \phi\right)\right|_{S_{0}}+ \\
\alpha_{1} \frac{\partial}{\partial x}\left(\left.\nabla S_{0} \cdot \nabla \phi_{b}\right|_{S_{0}}\right)+\alpha_{2} \frac{\partial}{\partial y}\left(\left.\nabla S_{0} \cdot \nabla \phi_{b}\right|_{S_{0}}\right)+\alpha_{3} \frac{\partial}{\partial z}\left(\left.\nabla S_{0} \cdot \nabla \phi_{b}\right|_{S_{0}}\right)+\cdots=0 .
\end{array}
$$

The unknown and time-dependent surface $S_{u}$ can also be expanded around the known surface $S_{0}$ by the Taylor expansion as [37]:

$$
S_{u}=\left(x^{\prime}-x\right) S_{x}+\left(y^{\prime}-y\right) S_{y}+\left(z^{\prime}-z\right) S_{z}=-\alpha_{1} S_{x}-\alpha_{2} S_{y}-\alpha_{3} S_{z}
$$

where for example the first derivative of the mean surface in the $\mathrm{x}$ direction $\frac{\partial S_{0}}{\partial x}$, is denoted here by $S_{x}$. If $S_{u}$ is inserted in to (B.8) then:

$$
\begin{aligned}
& -\dot{\alpha_{1}} S_{x}-\dot{\alpha_{2}} S_{y}-\dot{\alpha_{3}} S_{z}+S_{x} \phi_{x}+S_{y} \phi_{y}+S_{z} \phi_{z}+ \\
& \phi_{x}^{b}\left\{-\alpha_{1} S_{x x}-\alpha_{2} S_{x y}-\alpha_{3} S_{x z}-\Omega_{3} S_{y}+\Omega_{2} S_{z}\right\}+ \\
& \phi_{y}^{b}\left\{-\alpha_{1} S_{x y}-\alpha_{2} S_{y y}-\alpha_{3} S_{z y}+\Omega_{3} S_{x}-\Omega_{1} S_{z}\right\}+ \\
& \phi_{z}^{b}\left\{-\alpha_{1} S_{x z}-\alpha_{2} S_{y z}-\alpha_{3} S_{z z}-\Omega_{2} S_{x}+\Omega_{1} S_{y}\right\}+ \\
& \alpha_{1}\left\{S_{x} \phi_{x x}^{b}+S_{x x} \phi_{x}^{b}+S_{y} \phi_{x y}^{b}+S_{x y} \phi_{y}^{b}+S_{z} \phi_{x z}^{b}+S_{x z} \phi_{z}^{b}\right\}+ \\
& \alpha_{2}\left\{S_{x} \phi_{x y}^{b}+S_{x y} \phi_{x}^{b}+S_{y} \phi_{y y}^{b}+S_{y y} \phi_{y}^{b}+S_{z} \phi_{z y}^{b}+S_{y z} \phi_{z}^{b}\right\}+ \\
& \alpha_{3}\left\{S_{x} \phi_{x z}^{b}+S_{x z} \phi_{x}^{b}+S_{y} \phi_{y z}^{b}+S_{y z} \phi_{y}^{b}+S_{z} \phi_{z z}^{b}+S_{z z} \phi_{z}^{b}\right\}= \\
& -\dot{\alpha_{1}} S_{x}-\dot{\alpha_{2}} S_{y}-\dot{\alpha_{3}} S_{z}+S_{x} \phi_{x}+S_{y} \phi_{y}+S_{z} \phi_{z}+ \\
& \phi_{x}^{b}\left(-\Omega_{3} S_{y}+\Omega_{2} S_{z}\right)+\phi_{y}^{b}\left(-\Omega_{1} S_{z}+\Omega_{3} S_{x}\right)+\phi_{z}^{b}\left(-\Omega_{2} S_{x}+\Omega_{1} S_{y}\right)+ \\
& \alpha_{1}\left\{S_{x} \phi_{x x}^{b}+S_{y} \phi_{x y}^{b}+S_{z} \phi_{x z}^{b}\right\}+\alpha_{2}\left\{S_{x} \phi_{x y}^{b}+S_{y} \phi_{y y}^{b}+S_{z} \phi_{z y}^{b}\right\}+\alpha_{3}\left\{S_{x} \phi_{x z}^{b}+S_{y} \phi_{y z}^{b}+S_{z} \phi_{z z}^{b}\right\}=0 .
\end{aligned}
$$

As it has been defined by (A.6), the normal vector at the body surface is:

$$
\mathbf{n}=\frac{\left(S_{x}, S_{y}, S_{z}\right)}{|\nabla S|} .
$$


By this it is easy to verify that:

$$
\begin{gathered}
(\dot{\boldsymbol{\alpha}} \cdot \boldsymbol{n})|\nabla S|=\dot{\alpha}_{1} S_{x}+\dot{\alpha}_{2} S_{y}+\dot{\alpha}_{3} S_{z}, \\
\left(\frac{\partial \phi}{\partial n}\right)|\nabla S|=S_{x} \phi_{x}+S_{y} \phi_{y}+S_{z} \phi_{z}, \\
\left(-\left(\nabla \phi_{b} \cdot \nabla\right) \boldsymbol{\alpha} \cdot \boldsymbol{n}\right)|\nabla S|=S_{x}\left(\Omega_{3} \phi_{y}^{b}-\Omega_{2} \phi_{z}^{b}\right)+S_{y}\left(\Omega_{1} \phi_{z}^{b}-\Omega_{3} \phi_{x}^{b}\right)+S_{z}\left(\Omega_{2} \phi_{x}^{b}-\Omega_{1} \phi_{y}^{b}\right)+R, \\
\left((\boldsymbol{\alpha} \cdot \nabla) \nabla \phi_{b} \cdot \boldsymbol{n}\right)|\nabla S|= \\
S_{x}\left(\alpha_{1} \phi_{x x}^{b}+\alpha_{2} \phi_{x y}^{b}+\alpha_{3} \phi_{x z}^{b}\right)+S_{y}\left(\alpha_{1} \phi_{x y}^{b}+\alpha_{2} \phi_{y y}^{b}+\alpha_{3} \phi_{y z}^{b}\right)+S_{z}\left(\alpha_{1} \phi_{x z}^{b}+\alpha_{2} \phi_{z y}^{b}+\alpha_{3} \phi_{z z}^{b}\right)-R .
\end{gathered}
$$

Note that $R$ here denotes the set of all remaining terms which are identical in the last two relations, and will be cancelled. Finally the linearized boundary condition can be written as:

$$
\frac{\partial \phi}{\partial n}=\left\{\dot{\boldsymbol{\alpha}}+\left(\nabla \phi_{b} \cdot \nabla\right) \boldsymbol{\alpha}-(\boldsymbol{\alpha} \cdot \nabla) \nabla \phi_{b}\right\} \cdot \boldsymbol{n},
$$

which has been already derived by [18], and can be used both for the rigid and the flexible modes of the body motion. In the case of the rigid modes, the gradient of the displacement vector $\boldsymbol{\alpha}$, can be obtained easily. This allows us to identify the m-terms, as will be shown in the next section. For flexible modes, the gradient of the displacement vector $\boldsymbol{\alpha}$ is dependent on the desired shape of the flexible body, and a body boundary condition expressed using generalized $\mathrm{m}$-terms is also obtained in section B.4.

\section{B.3 Rigid-body m-terms}

For the six rigid modes of motion, the linearized body boundary condition can be expressed using the well-known m-terms [38]. This is achieved through a re-arrangement of the boundary condition to get the following terms:

$$
\begin{aligned}
& \{\dot{\boldsymbol{\xi}} \cdot \boldsymbol{n}+\dot{\boldsymbol{\Omega}} \cdot(\boldsymbol{r} \times \boldsymbol{n})\}|\nabla S|= \\
& \dot{\xi_{1}} S_{x}+\dot{\xi}_{2} S_{y}+\dot{\xi}_{3} S_{z}+\dot{\Omega}_{1}\left(y S_{z}-z S_{y}\right)+\dot{\Omega}_{2}\left(z S_{x}-x S_{z}\right)+\dot{\Omega}_{3}\left(x S_{y}-y S_{x}\right), \\
& \left\{\boldsymbol{\xi} \cdot\left(-(\boldsymbol{n} \cdot \nabla) \nabla \phi_{b}\right)\right\}|\nabla S|= \\
& \xi_{1}\left\{S_{x} \phi_{x x}^{b}+S_{y} \phi_{x y}^{b}+S_{z} \phi_{x z}^{b}\right\}+\xi_{2}\left\{S_{x} \phi_{x y}^{b}+S_{y} \phi_{y y}^{b}+S_{z} \phi_{y z}^{b}\right\}+\xi_{3}\left\{S_{x} \phi_{x z}^{b}+S_{y} \phi_{y z}^{b}+S_{z} \phi_{z z}^{b}\right\}, \\
& \left\{\boldsymbol{\Omega} \cdot\left(-(\boldsymbol{n} \cdot \nabla)\left(\boldsymbol{r} \times \nabla \phi_{b}\right)\right)\right\}|\nabla S|= \\
& \Omega_{1}\left\{S_{x}\left(y \phi_{x z}^{b}-z \phi_{x y}^{b}\right)+S_{y}\left(\phi_{z}^{b}+y \phi_{y z}^{b}-z \phi_{y y}^{b}\right)+S_{z}\left(y \phi_{z z}^{b}-\phi_{y}^{b}-z \phi_{y z}^{b}\right)\right\}+ \\
& \Omega_{2}\left\{S_{x}\left(z \phi_{x x}^{b}-\phi_{z}^{b}-x \phi_{x z}^{b}\right)+S_{y}\left(z \phi_{x y}^{b}-x \phi_{y z}^{b}\right)+S_{z}\left(\phi_{x}^{b}+z \phi_{x z}^{b}-x \phi_{z z}^{b}\right)\right\}+ \\
& \Omega_{3}\left\{S_{x}\left(\phi_{y}^{b}+x \phi_{x y}^{b}-y \phi_{x x}^{b}\right)+S_{y}\left(x \phi_{y y}^{b}-\phi_{x}^{b}-y \phi_{x y}^{b}\right)+S_{z}\left(x \phi_{y z}^{b}-y \phi_{x z}^{b}\right)\right\} .
\end{aligned}
$$

So the body boundary condition can be written as:

$$
\frac{\partial \phi}{\partial n}=\dot{\boldsymbol{\xi}} \cdot \boldsymbol{n}+\dot{\boldsymbol{\Omega}} \cdot(\boldsymbol{r} \times \boldsymbol{n})+\boldsymbol{\xi} \cdot\left(-(\boldsymbol{n} \cdot \nabla) \nabla \phi_{b}\right)+\boldsymbol{\Omega} \cdot\left(-(\boldsymbol{n} \cdot \nabla)\left(\boldsymbol{r} \times \nabla \phi_{b}\right)\right),
$$


which is in fact the condition for a motion comprised of all 6 degrees of freedom. But for the $k$ th mode of the body motion this condition could be expressed in a more compact form using the $m$-terms as follows:

$$
\frac{\partial \phi_{k}}{\partial n}=\dot{\xi}_{k} n_{k}+\xi_{k} m_{k}, \quad k=1, \cdots 6,
$$

in which:

$$
\begin{aligned}
\left(\xi_{1}, \xi_{2}, \xi_{3}\right) & =\left(\xi_{1}, \xi_{2}, \xi_{3}\right), \\
\left(\xi_{4}, \xi_{5}, \xi_{6}\right) & =\left(\Omega_{1}, \Omega_{2}, \Omega_{3}\right), \\
\left(n_{1}, n_{2}, n_{3}\right) & =\boldsymbol{n}, \\
\left(n_{4}, n_{5}, n_{6}\right) & =(\boldsymbol{r} \times \boldsymbol{n}) \\
\left(m_{1}, m_{2}, m_{3}\right) & =-(\boldsymbol{n} \cdot \nabla) \nabla \phi_{b} \\
\left(m_{4}, m_{5}, m_{6}\right) & =-(\boldsymbol{n} \cdot \nabla)\left(\boldsymbol{r} \times \nabla \phi_{b}\right) .
\end{aligned}
$$

Or in the expanded form:

$$
\begin{aligned}
& \left(m_{1}, m_{2}, m_{3}\right)=-\left(n_{1} \partial_{x}+n_{2} \partial_{y}+n_{3} \partial_{z}\right)\left(\phi_{x}^{b}, \phi_{y}^{b}, \phi_{z}^{b}\right) \\
& \left(m_{4}, m_{5}, m_{6}\right)=-\left(n_{1} \partial_{x}+n_{2} \partial_{y}+n_{3} \partial_{z}\right)\left(y \phi_{z}^{b}-z \phi_{y}^{b}, z \phi_{x}^{b}-x \phi_{z}^{b}, x \phi_{y}^{b}-y \phi_{x}^{b}\right) .
\end{aligned}
$$

\section{B.4 Generalized m-terms}

According to [39], for the $k$ th flexible mode of the floating body a time-independent vector shape function can be defined as follows:

$$
\boldsymbol{F}_{k}(x, y, z)=\left(u_{k}, v_{k}, w_{k}\right),
$$

which describes the displacement of the body in the corresponding $\mathrm{x}, \mathrm{y}$ and $\mathrm{z}$ direction. In the time domain the generalized motion of the body in $k$ th mode can be expressed by:

$$
\boldsymbol{\xi}_{k}(x, y, z, t)=\xi_{k}(t) \boldsymbol{F}_{k}(x, y, z) .
$$

The time-dependent amplitude of the motion in $k$ th mode is related to the corresponding complex phasor $\hat{\xi}_{k}$ in the frequency domain as:

$$
\xi_{k}(t)=\operatorname{Re}\left\{\hat{\xi}_{k} e^{-i \omega_{e} t}\right\}
$$

where $\omega_{e}$ is the wave encounter frequency. The above mentioned displacement vector is generalized and includes also the rigid modes with:

$$
\begin{array}{ll}
\boldsymbol{F}_{1}=(1,0,0), & \boldsymbol{F}_{4}=(0,-z, y), \\
\boldsymbol{F}_{2}=(0,1,0), & \boldsymbol{F}_{5}=(z, 0,-x), \\
\boldsymbol{F}_{3}=(0,0,1), & \boldsymbol{F}_{6}=(-y, x, 0),
\end{array}
$$

in which $\boldsymbol{F}_{k}$ for $\mathrm{k}=4,5$ and 6 is computed as [39]:

$$
\boldsymbol{F}_{k}=\boldsymbol{F}_{j-3} \times \boldsymbol{r} .
$$

Note that the divergence of the displacement vectors for the rigid modes are all 0 , but this is not true generally for the flexible modes. The displacement vector from (B.17) is inserted in to the linearized 
body boundary condition (B.11) to obtain the body boundary condition for the $k$ th generalized mode as:

$$
\begin{aligned}
\frac{\partial \phi_{k}}{\partial n} & =\left(\dot{\xi}_{k} \boldsymbol{F}_{k}+\xi_{k}\left(\nabla \phi_{b} \cdot \nabla\right) \boldsymbol{F}_{k}-\xi_{k}\left(\boldsymbol{F}_{k} \cdot \nabla\right) \nabla \phi_{b}\right) \cdot \boldsymbol{n}, \\
& =\dot{\xi}_{k} n_{k}+\xi_{k} m_{k}
\end{aligned}
$$

where:

$$
\begin{aligned}
n_{k} & =\boldsymbol{F}_{k} \cdot \boldsymbol{n}=\sum_{j=1}^{3} F_{k}^{j} n_{j}, \\
m_{k} & =\left(\left(\nabla \phi_{b} \cdot \nabla\right) \boldsymbol{F}_{k}-\left(\boldsymbol{F}_{k} \cdot \nabla\right) \nabla \phi_{b}\right) \cdot \boldsymbol{n} .
\end{aligned}
$$

Note that $j$ th component of the displacement vector is given by $F_{k}^{j}$. For more clarification the generalized $\mathrm{m}$-term are expanded as follows:

$$
\begin{aligned}
m_{k}= & n_{1}\left(\phi_{x}^{b} F_{k x}^{1}+\phi_{y}^{b} F_{k y}^{1}+\phi_{z}^{b} F_{k z}^{1}-F_{k}^{1} \phi_{x x}^{b}-F_{k}^{2} \phi_{x y}^{b}-F_{k}^{3} \phi_{x z}^{b}\right)+ \\
& n_{2}\left(\phi_{x}^{b} F_{k x}^{2}+\phi_{y}^{b} F_{k y}^{2}+\phi_{z}^{b} F_{k z}^{2}-F_{k}^{1} \phi_{x y}^{b}-F_{k}^{2} \phi_{y y}^{b}-F_{k}^{3} \phi_{y z}^{b}\right)+ \\
& n_{3}\left(\phi_{x}^{b} F_{k x}^{3}+\phi_{y}^{b} F_{k y}^{3}+\phi_{z}^{b} F_{k z}^{3}-F_{k}^{1} \phi_{x z}^{b}-F_{k}^{2} \phi_{y z}^{b}-F_{k}^{3} \phi_{z z}^{b}\right),
\end{aligned}
$$

in which for example the third component of the $y$ derivative of the $k$ th mode displacement vector is denoted by $F_{k y}^{3}$. It is straitforward to verify that the m-terms from (B.14) and (B.15) can be in fact recovered from the above mentioned generalized equation. For instance for the sway rigid mode $k=2, \boldsymbol{F}_{2}=(0,1,0)$, and the corresponding m-term using (B.23) is obtained as:

$$
m_{2}=-\left(n_{1} \phi_{x y}^{b}+n_{2} \phi_{y y}^{b}+n_{3} \phi_{y z}^{b}\right) .
$$

Or for the pitch mode $k=5$, where $\boldsymbol{F}_{5}=(z, 0,-x), F_{5 z}^{1}=-F_{5 x}^{3}=1$ and all other derivatives of the displacement vector are equal to zero, the $m_{5}$ terms becomes:

$$
m_{5}=-n_{1}\left(z \phi_{x x}^{b}-x \phi^{b} x z+\phi_{z}^{b}\right)-n_{2}\left(z \phi_{x y}^{b}-x \phi_{z y}^{b}\right)-n_{3}\left(z \phi_{x z}^{b}-x \phi^{b} z z+\phi_{x}^{b}\right) .
$$

Note that if the uniform flow is subtracted form the base flow as is shown in (2.4), the equation for the m-terms is obtained by replacing $\phi_{b}$ with $\phi_{b}^{\prime}$ and $\phi_{x}^{b}$ with $-U+\phi_{x}^{\prime b}$ in (B.23), (B.14) or (B.15). 